TRANSACTIONS OF THE

AMERICAN MATHEMATICAL SOCIETY

Volume 365, Number 12, December 2013, Pages 6595-6623

S 0002-9947(2013)05929-6

Article electronically published on May 14, 2013

\title{
SYMPLECTIC BRANCHING LAWS AND HERMITIAN SYMMETRIC SPACES
}

\author{
BENJAMIN SCHWARZ AND HENRIK SEPPÄNEN
}

\begin{abstract}
Let $G$ be a complex simple Lie group, and let $U \subseteq G$ be a maximal compact subgroup. Assume that $G$ admits a homogenous space $X=G / Q=$ $U / K$ which is a compact Hermitian symmetric space. Let $\mathscr{L} \rightarrow X$ be the ample line bundle which generates the Picard group of $X$. In this paper we study the restrictions to $K$ of the family $\left(H^{0}\left(X, \mathscr{L}^{k}\right)\right)_{k \in \mathbb{N}}$ of irreducible $G$ representations. We explicitly describe the moment polytopes for the moment maps $X \rightarrow \mathfrak{k}^{*}$ associated to positive integer multiples of the Kostant-Kirillov symplectic form on $X$, and we use these, together with an explicit characterization of the closed $K^{\mathbb{C}}$-orbits on $X$, to find the decompositions of the spaces $H^{0}\left(X, \mathscr{L}^{k}\right)$. We also construct a natural Okounkov body for $\mathscr{L}$ and the $K$ action, and we identify it with the smallest of the moment polytopes above. In particular, the Okounkov body is a convex polytope. In fact, we even prove the stronger property that the semigroup defining the Okounkov body is finitely generated.
\end{abstract}

\section{INTRODUCTION}

In this paper we consider the following setting. Let $G$ be a complex simple Lie group, and assume that $G$ admits a quotient $X:=G / Q$ which is a compact Hermitian symmetric space. Then $Q$ is a maximal parabolic subgroup. Moreover, we can write $X$ as $X=U / K$, where $U \subseteq G$ is a maximal compact subgroup, and $K:=U \cap Q$. The Picard group of $X$, which is isomorphic to the group of holomorphic characters $Q \rightarrow \mathbb{C}^{\times}$, is $\mathbb{Z}$. Let $\mathscr{L} \rightarrow X$ be the ample generator for the Picard group. We are concerned with the decomposition under $K$ of the irreducible $G$-representations given by the family $H^{0}\left(X, \mathscr{L}^{k}\right)$, where $k \in \mathbb{N}$.

In order to put our approach to the decomposition problem into its proper framework, we make a small digression into a more general setting. For a more thorough treatment we refer to $\mathrm{Sj} 95$ and the references therein. We now temporarily let $K$ denote an arbitrary compact Lie group and assume that $K$ acts holomorphically and in a Hamiltonian fashion on the connected compact Kähler manifold $(M, \omega)$ with moment map $\tau: M \rightarrow \mathfrak{k}^{*}$. Assume that $(M, \omega)$ admits a prequantum line bundle $\mathcal{L}$. The action of $K$ then lifts to an action on $\mathcal{L}$, and hence we have a representation of $K$ on the space $H^{0}(M, \mathcal{L})$. One may then ask how

Received by the editors November 28, 2011 and, in revised form, May 7, 2012 and August 2, 2012 .

2010 Mathematics Subject Classification. Primary 22E46; Secondary 53D20, 17C50, 32M15, $32 \mathrm{~L} 05$.

Key words and phrases. Branching law, holomorphic line bundle, Hermitian symmetric space, moment map, Jordan pair, Okounkov body.

The second author was supported by the DFG Priority Programme 1388 Representation Theory.

C) 2013 American Mathematical Society Reverts to public domain 28 years from publication 
$H^{0}(M, \mathcal{L})$ decomposes under $K$. For this purpose it is useful to realize the irreducible $K$-representation of highest weight $\xi$ as the space of holomorphic sections $H^{0}\left(\mathcal{O}_{\xi}^{K}, \mathscr{L}_{\xi}\right)$, where $\mathcal{O}_{\xi}^{K} \subseteq \mathfrak{k}^{*}$ is the coadjoint orbit through $\xi$ and $\mathscr{L}_{\xi}$ is the prequantum line bundle attached to the Kostant-Kirillov symplectic form on $\mathcal{O}_{\xi}^{K}$. The multiplicity of the representation $H^{0}\left(\mathcal{O}_{\xi}^{K}, \mathscr{L}_{\xi}\right)$ in $H^{0}(M, \mathcal{L})$ is given by the dimension of the space, $H^{0}\left(M \times \overline{\mathcal{O}_{\xi}^{K}}, \mathcal{L} \otimes \overline{\mathscr{L}_{\xi}^{*}}\right)^{K}$, of $K$-invariant holomorphic sections of the line bundle $\mathcal{L} \otimes \overline{\mathscr{L}_{\xi}^{*}} \rightarrow M \times \overline{\mathcal{O}_{\xi}^{K}}\left(\left[\right.\right.$ GS82, Thm. 6.2]). Here $\overline{\mathcal{O}_{\xi}^{K}}$ denotes the topological space $\mathcal{O}_{\xi}^{K}$ equipped with the reverse complex structure and the symplectic form given by -1 times the Kostant-Kirillov form. The fibre over $x \in \mathcal{O}_{\xi}^{K}$ of the line bundle $\overline{\mathscr{L}_{\xi}^{*}}$ consists of the space of antilinear complex-valued functionals on $\left(\mathscr{L}_{\xi}\right)_{x}$ (cf. GS82]). The group $K$ now acts holomorphically and in a Hamiltonian fashion on $M \times \overline{\mathcal{O}_{\xi}^{K}}$ with moment map $\tau^{\xi}: M \times \overline{\mathcal{O}_{\xi}^{K}} \rightarrow \mathfrak{k}^{*}$ given by $\tau^{\xi}(m, f):=\tau(m)-f$.

An obvious question is whether the space $H^{0}\left(M \times \overline{\mathcal{O}_{\xi}^{K}}, \mathcal{L} \otimes \overline{\mathscr{L}_{\xi}^{*}}\right)^{K}$ can be interpreted as the space of all holomorphic sections of some line bundle over some "quotient" of $M \times \overline{\mathcal{O}_{\xi}^{K}}$ by $K$. Indeed, this holds for the Mumford quotient

$$
\left(M \times \overline{\mathcal{O}_{\xi}^{K}}\right)_{0}:=\left(M \times \overline{\mathcal{O}_{\xi}^{K}}\right)_{s s} / / K^{\mathbb{C}},
$$

where $\left(M \times \overline{\mathcal{O}_{\xi}^{K}}\right)_{s s}$ is the open subset consisting of the semistable points of $(M \times$ $\left.\overline{\mathcal{O}_{\xi}^{K}}\right)$. An interesting feature, and one which links the Mumford quotient to the symplectic geometry, is that $\left(M \times \overline{\mathcal{O}_{\xi}^{K}}\right)_{0}$ is homeomorphic to the topological quotient $\left(\tau^{\xi}\right)^{-1}(0) / K$ (cf. Sj95, Thm. 2.5]), the symplectic reduction at 0 . Moreover, the following result holds.

Theorem 1.1 ([Sj95, Corollary 1]). If $\xi$ does not lie in the image $\tau(M)$, then the irreducible representation of highest weight $\xi$ does not occur in $H^{0}(M, \mathcal{L})$.

The space $\left(M \times \overline{\mathcal{O}_{\xi}^{K}}\right)_{0}$ also carries the structure of a complex projective variety. In fact, it is isomorphic to $\operatorname{Proj}\left(\oplus_{k=0}^{\infty} H^{0}\left(M \times \overline{\mathcal{O}_{\xi}^{K}},\left(\mathcal{L} \otimes \overline{\mathscr{L}_{\xi}^{*}}\right)^{k}\right)^{K}\right)$. Moreover, for large enough $q$, the line bundle $\mathcal{L}^{q} \otimes\left(\overline{\mathscr{L}_{\xi}^{*}}\right)^{q}$ induces a line bundle $\left(\mathcal{L}^{q} \otimes\left(\overline{\mathscr{L}_{\xi}^{*}}\right)^{q}\right)_{0}$ over $\left(M \times \overline{\mathcal{O}_{\xi}^{K}}\right)_{s s} / / K^{\mathbb{C}}$, the total space of which is $\left(\left.\left(\mathcal{L} \otimes \overline{\mathscr{L}_{\xi}^{*}}\right)^{q}\right|_{\left(M \times \overline{\mathcal{O}_{\xi}^{K}}\right)_{s s}}\right) / K^{\mathbb{C}}$. For such $q$, there is an isomorphism

$$
H^{0}\left(M \times \overline{\mathcal{O}_{\xi}^{K}},\left(\mathcal{L} \otimes \overline{\mathscr{L}_{\xi}^{*}}\right)^{q}\right)^{K} \cong H^{0}\left(\left(M \times \overline{\mathcal{O}_{\xi}^{K}}\right)_{0},\left(\mathcal{L}^{q} \otimes\left(\overline{\mathscr{L}_{\xi}^{*}}\right)^{q}\right)_{0}\right) .
$$

Under favourable conditions, e.g. the vanishing of all cohomology groups $H^{i}((M \times$ $\left.\left.\overline{\mathcal{O}_{\xi}^{K}}\right)_{0},\left(\mathcal{L}^{k q} \otimes\left(\overline{\mathscr{L}_{\xi}^{*}}\right)^{k q}\right)_{0}\right)$, for $k>0$ and $i>0$, the asymptotics of $\operatorname{dim} H^{0}((M \times$ $\left.\left.\overline{\mathcal{O}_{\xi}^{K}}\right)_{0},\left(\mathcal{L}^{k q} \otimes\left(\overline{\mathscr{L}_{\xi}^{*}}\right)^{k q}\right)_{0}\right)$ as $k \rightarrow \infty$ is given by the Riemann-Roch theorem for (singular) complete schemes (cf. [Fu98, Example 18.3.6]).

Although this machinery works well in principle, a major obstruction to applying it in particular cases in order to obtain explicit asymptotic expressions for multiplicities is that the moment maps $\tau^{\xi}$, and hence their fibres, are in general notoriously hard to compute.

In this paper we are able to compute the moment map $\mu_{\mathfrak{k}}: X \rightarrow \mathfrak{k}^{*}$ for the $K$ action by using an explicit Jordan-theoretic description of $X$. We also prove that for any $\nu \in \mu_{\mathfrak{k}}(X)$, the stabilizer, $K_{\nu}$, of $\nu$ acts transitively on the fibre $\mu_{\mathfrak{k}}^{-1}(\nu)$. As 
a consequence, the decomposition of $H^{0}\left(X, \mathscr{L}^{k}\right)$ under $K$ is multiplicity free for every $k \in \mathbb{N}$. We also explicitly describe the moment polytope for $k \mu_{\mathfrak{k}}$ for any $k \in \mathbb{N}$, i.e., the intersection of $k \mu_{\mathfrak{k}}(X)$ with a closed Weyl chamber, as well as the integral points in the moment polytope. By Theorem 1.1. these are the only weights that can occur as highest weights of irreducible $K$-representations in $H^{0}\left(X, \mathscr{L}^{k}\right)$. We prove that all these integral points in fact do occur. In fact, from the particular form of the integral weights in the moment polytopes for the $k \mu_{\mathfrak{k}}$ it turns out that it suffices to prove this for $k=1$, i.e., that all the integral points in the moment polytope for $\mu_{\mathfrak{k}}$ parameterize irreducible $K$-representations in $H^{0}(X, \mathscr{L})$.

In the special case $k=1$ we prove that the integral points stand in a one-to-one correspondence with the closed $K^{\mathbb{C}}$-orbits, $X_{0}, \ldots, X_{r}$, in $X$. The number $r$ is the rank of $X$ as a symmetric space. We also give a Jordan-theoretic characterization of these orbits. Using this characterization, we give a geometric decomposition of $H^{0}(X, \mathscr{L})$ under $K$. The $K$-equivariant embedding $W_{i} \rightarrow H^{0}(X, \mathscr{L})$ of the irreducible representation $W_{i}$ corresponding to the orbit $X_{i}$ is a section for the restriction map $H^{0}(X, \mathscr{L}) \rightarrow H^{0}\left(X_{i},\left.\mathscr{L}\right|_{X_{i}}\right)$.

We also define an Okounkov body for the line bundle $\mathscr{L}$ and the $K$-action (cf. Ok96]) by using a canonical local trivialization of sections. The semigroup defining the Okounkov body describes the initial monomial terms of the polynomials that are local trivializations of highest weight vectors for irreducible $K$-subrepresentations. Using the decompositions for the spaces $H^{0}\left(X, \mathscr{L}^{k}\right)$ under $K$ we are able to prove that this semigroup is finitely generated.

We would like to point out that the decomposition of the spaces $H^{0}\left(X, \mathscr{L}^{k}\right)$ under the group $K$ can probably be proved in an easier and quite straightforward way, e.g. using the main result in Sc69]. Rather, our interest lies in the method of proof, namely in testing how explicit results are that one can obtain by the study of moment maps and symplectic reduction. Finally, we would like to remark that the multiplicity-freeness result can also be derived using the notion of visible actions developed by Kobayashi (cf. [Ko08, Thm. E]).

The paper is organized as follows. In Section 2 and Section 3 we recall the preliminaries from Lie theory and Jordan theory, respectively. In Section 4 we study the local trivializations of holomorphic sections and use these to define an Okounkov body for $\mathscr{L}$ and the $K$-action. Section 5 is devoted to closed $K^{\mathbb{C}}$-orbits in $X$ and a geometric decomposition theorem. In Section 6 we describe the moment polytope and the symplectic reductions. In Section 7 we prove the decomposition theorem for $H^{0}\left(X, \mathscr{L}^{k}\right)$ using the results from previous sections. In Section 8 we use the results from Section 7 to identify the Okounkov body with the moment polytope for $\mu_{\mathfrak{k}}$.

\section{Preliminaries from Lie theory}

Let $\mathfrak{g}$ be a complex simple Lie algebra, and let $\mathfrak{h}$ be a fixed Cartan subalgebra of $\mathfrak{g}$. Let $\Phi=\Phi(\mathfrak{g}, \mathfrak{h})$ be the set of roots with respect to $\mathfrak{h}$. Let $\Phi^{+} \subseteq \Phi$ be a positive system and $\left\{\beta_{1}, \ldots, \beta_{s}\right\}$ the corresponding simple roots. For every $\alpha \in \Phi^{+}$, fix an $\mathfrak{s l}_{2}$-triple $\left\{E_{\alpha}, H_{\alpha}, E_{-\alpha}\right\}$ with $H_{\alpha} \in \mathfrak{h}, E_{\alpha} \in \mathfrak{g}_{\alpha}$, and $E_{-\alpha} \in \mathfrak{g}_{-\alpha}$, normalized so that the identitiy

$$
\left[E_{\alpha}, E_{\beta}\right]=N_{\alpha, \beta} E_{\alpha+\beta}
$$


holds for all $\alpha, \beta \in \Phi$ such that $\alpha+\beta \in \Phi$, with constants $N_{\alpha, \beta} \in \mathbb{R}$ satisfying $N_{-\alpha,-\beta}=-N_{\alpha, \beta}$. Also, set $F_{\alpha}:=E_{-\alpha}$ for $\alpha \in \Phi^{+}$. Let

$$
\mathfrak{u}:=\bigoplus_{j=1}^{s} i H_{\beta_{j}} \oplus \bigoplus_{\alpha \in \Phi^{+}} \mathbb{R}\left(E_{\alpha}-F_{\alpha}\right) \oplus \bigoplus_{\alpha \in \Phi^{+}} \mathbb{R} i\left(E_{\alpha}+F_{\alpha}\right)
$$

be the canonical compact real form of $\mathfrak{g}$ and $\theta: \mathfrak{g} \rightarrow \mathfrak{g}$ be the associated Cartan involution of $\mathfrak{g}$. Let

$$
\mathfrak{t}:=\bigoplus_{j=1}^{s} i H_{\beta_{j}} \subseteq \mathfrak{u}
$$

be the maximal abelian subalgebra of $\mathfrak{u}$ with $\mathfrak{h}^{\theta}=\mathfrak{t}$. Define

$$
\mathfrak{n}^{+}:=\bigoplus_{\alpha \in \Phi^{+}} \mathfrak{g}_{\alpha}, \quad \mathfrak{n}^{-}:=\bigoplus_{\alpha \in-\Phi^{+}} \mathfrak{g}_{\alpha}
$$

and let

$$
\mathfrak{b}:=\mathfrak{h} \oplus \mathfrak{n}^{+}
$$

be the Borel subalgebra defined by the positive system $\Phi^{+}$. Then $\mathfrak{g}$ can be decomposed as

$$
\mathfrak{g}=\mathfrak{b} \oplus \mathfrak{n}^{-} .
$$

For a root, $\alpha$, let $m_{\beta_{i}}(\alpha)$ be the multiplicity of $\beta_{i}$ in $\alpha$, i.e., $\alpha=\sum_{i=1}^{s} m_{\beta_{i}}(\alpha) \beta_{i}$.

The condition that a complex simple Lie group $G$ admits a quotient $G / Q$ which is a compact Hermitian symmetric space is equivalent to the condition that the Lie algebra of $G$ admits a simple root which has multiplicity at most one in every positive root. We therefore assume that $\beta_{1}$ is such a simple root. We define subsets of $\Phi$ by

$$
\Phi_{Q}:=\left\{\alpha \in \Phi \mid m_{\beta_{1}}(\alpha) \geq 0\right\}, \quad \Phi_{L}:=\left\{\alpha \in \Phi \mid m_{\beta_{1}}(\alpha)=0\right\},
$$

and Lie subalgebras

$$
\mathfrak{l}:=\mathfrak{h} \oplus \bigoplus_{\alpha \in \Phi_{L}} \mathfrak{g}_{\alpha}, \quad \mathfrak{q}:=\mathfrak{h} \oplus \bigoplus_{\alpha \in \Phi_{Q}} \mathfrak{g}_{\alpha}
$$

We also define

$$
\mathfrak{p}^{+}:=\bigoplus_{\alpha \in \Phi_{Q} \backslash \Phi_{L}} \mathfrak{g}_{\alpha}, \quad \mathfrak{p}^{-}:=\bigoplus_{\alpha \in \Phi_{Q} \backslash \Phi_{L}} \mathfrak{g}_{-\alpha}
$$

Then we have

$$
\mathfrak{q}=\mathfrak{l} \oplus \mathfrak{p}^{+}
$$

The Lie algebra $\mathfrak{q}$ is a maximal parabolic subalgebra of $\mathfrak{g}$ containing $\mathfrak{b}$, and $\mathfrak{p}^{+}$and $\mathfrak{l}$ are the nilpotent radical of $\mathfrak{q}$ and the Levi factor of $\mathfrak{q}$, respectively. From the assumption that $m_{\beta_{1}}(\alpha) \in\{-1,0,1\}$ for any root $\alpha$, it readily follows that $\mathfrak{p}^{+}$is an abelian subalgebra.

The Lie algebra $\mathfrak{l}$ is reductive with semisimple part

$$
\mathfrak{l}^{\prime}:=[\mathfrak{l}, \mathfrak{l}]=\mathfrak{h}_{L} \oplus \underset{\alpha \in \Phi_{L}}{\bigoplus} \mathfrak{g}_{\alpha}
$$

where

$$
\mathfrak{h}_{L}:=H_{\beta_{2}} \oplus \cdots \oplus H_{\beta_{s}}
$$

is a Cartan subalgebra of $\mathfrak{l}^{\prime}$. Then $\Phi_{L}$ is the set of roots of $\mathfrak{l}$ with respect to $\mathfrak{h}_{L}$. The set $\Phi_{L}^{+}:=\Phi_{L} \cap \Phi^{+}$is a positive system in $\Phi_{L}$, and we accordingly define the subalgebras

$$
\mathfrak{n}_{L}^{+}:=\bigoplus_{\alpha \in \Phi_{L}^{+}} \mathfrak{l}_{\alpha}, \quad \mathfrak{n}_{L}^{-}:=\bigoplus_{\alpha \in-\Phi_{L}^{+}} \mathfrak{l}_{\alpha}
$$


of $\mathfrak{l}$. The subalgebra $\mathfrak{l}$ is obviously $\theta$-invariant, and we set

$$
\mathfrak{k}:=\mathfrak{l}^{\theta}=\mathfrak{l} \cap \mathfrak{u} .
$$

Let $\zeta_{0} \in \mathfrak{k}$ be a basis vector for $\mathfrak{z}(\mathfrak{l})$, the centre of $\mathfrak{l}$. Then

$$
\mathfrak{l}=\mathfrak{l}^{\prime} \oplus \mathbb{C} \zeta_{0} .
$$

We now return to the roots in $\Phi^{+}$and construct a particular numbering of them. Equip the root lattice with the lexicographic order coming from the identification with the lattice $\mathbb{Z} \beta_{1} \oplus \cdots \oplus \mathbb{Z} \beta_{s}$. Then $\beta_{1}>\ldots>\beta_{s}$. Moreover, $\alpha>\gamma$, for any $\alpha \in \Phi_{Q} \backslash \Phi_{L}$ and $\gamma \in \Phi_{L}$.

We construct a maximal set $\left\{\gamma_{1}, \ldots, \gamma_{r}\right\} \subseteq \Phi_{Q} \backslash \Phi_{L}$ of strongly orthogonal roots, i.e., the $\gamma_{i}$ satisfy the property that for all pairs $\left\{\gamma_{i}, \gamma_{j}\right\}$ is neither $\gamma_{i}+\gamma_{j}$ nor $\gamma_{i}-\gamma_{j}$ a root.

First, put $\gamma_{1}:=\beta_{1}$. Assuming that $\gamma_{1}, \ldots, \gamma_{i}$ are defined, let $\gamma_{i+1}$ be the smallest root in $\Phi_{Q} \backslash \Phi_{L}$ such that $\gamma_{j} \pm \gamma_{i+1} \notin \Phi$ for $j=1, \ldots, i$.

Given the roots $\gamma_{1}, \ldots, \gamma_{r}$, we now consider a particular decomposition of the maximal abelian subalgebra $\mathfrak{t} \subseteq \mathfrak{k}$. Let

$$
\kappa: \mathfrak{g} \times \mathfrak{g} \rightarrow \mathbb{C}
$$

be the Killing form of $\mathfrak{g}$. Put $\mathfrak{s}^{\prime}:=\mathbb{R} i H_{\gamma_{1}} \oplus \cdots \oplus \mathbb{R} i H_{\gamma_{r}} \subseteq \mathfrak{t}$, and consider the decomposition $\mathfrak{t}=\mathfrak{s}^{\prime} \oplus\left(\mathfrak{s}^{\prime}\right)^{\perp}$, where $\left(\mathfrak{s}^{\prime}\right)^{\perp} \subseteq \mathfrak{t}$ is the orthogonal complement to $\mathfrak{s}^{\prime}$ with respect to the restriction of $\kappa$ to $t$. Notice that

$$
\left(\mathfrak{s}^{\prime}\right)^{\perp}=\left\{H \in \mathfrak{t} \mid \gamma_{i}(H)=0, \quad i=1, \ldots, r\right\} .
$$

With respect to this decomposition of $\mathfrak{t}$, let $\zeta \in \mathfrak{t}$ denote the $\left(\mathfrak{s}^{\prime}\right)^{\perp}$-component of $\zeta_{0}$. Now put $\mathfrak{s}:=\mathfrak{s}^{\prime} \oplus \mathbb{R} \zeta$, and consider the decomposition

$$
\mathfrak{t}=\mathfrak{s} \oplus(\mathfrak{s})^{\perp},
$$

where $(\mathfrak{s})^{\perp} \subseteq \mathfrak{t}$ is the orthogonal complement to $\mathfrak{s}$ with respect to the restriction of $\kappa$ to $\mathrm{t}$.

We now enumerate the roots in $\Phi_{Q} \backslash \Phi_{L}$ as follows. First, let $\alpha_{1}:=\gamma_{1}, \ldots, \alpha_{r}:=\gamma_{r}$. Then, let $\alpha_{r+1}, \ldots, \alpha_{n}$ be the remaining roots in $\Phi_{Q} \backslash \Phi_{L}$, numbered in such a way that

$$
\alpha_{i}<\alpha_{j} \text { for all } r+1 \leq i<j \leq n
$$

(with respect to the lexicographic order above). The vectors $F_{1}:=F_{\alpha_{1}}, \ldots, F_{n}:=$ $F_{\alpha_{n}}$ form a basis for $\mathfrak{p}^{-}$.

We now shift our focus to the level of Lie groups. Let $G$ be a 1-connected complex Lie group with Lie algebra $\mathfrak{g}$. Define the subgroups $B:=N_{G}(\mathfrak{b}), Q:=N_{G}(\mathfrak{q})$, $L:=N_{G}\left(\mathfrak{p}^{-}\right) \cap N_{G}(\mathfrak{l}) \cap N_{G}\left(\mathfrak{p}^{+}\right)$with Lie algebras $\mathfrak{b}, \mathfrak{q}$, and $\mathfrak{l}$, respectively. Let $P^{-}:=\left\langle\exp \mathfrak{p}^{-}\right\rangle$be the integral subgroup of $G$ generated by $\mathfrak{p}^{-}$. Moreover, we put $B_{L}:=N_{L}\left(\mathfrak{h} \oplus \mathfrak{n}_{L}^{+}\right) \subseteq L$. Then $B_{L}$ is a Borel subgroup of $L$. Also, let $N_{L}^{+}$and $N_{L}^{-}$be the integral subgroups of $L$ with Lie algebras $\mathfrak{n}_{L}^{+}$and $\mathfrak{n}_{L}^{-}$, respectively.

We will be concerned with the homogeneous space $X:=G / Q$. From the point of view of symplectic geometry it is convenient to describe $X$ as a homogeneous space under a compact Lie group. For this purpose, let $U:=\exp \langle\mathfrak{u}\rangle \subseteq G$ be the integral subgroup of $G$ generated by $\mathfrak{u}$. Then, since $G / Q$ is connected, we have

$$
X=G / Q=U / K,
$$


where

$$
K:=Q \cap U
$$

Notice that $K$ has Lie algebra $\mathfrak{k}$.

We will now take a closer look at a particular choice of local coordinates for $X$. The multiplication map

$$
P^{-} \times Q \rightarrow G, \quad(p, q) \mapsto p q
$$

is holomorphic and injective with open image. Moreover, the exponential map

$$
\exp : \mathfrak{p}^{-} \rightarrow P^{-}
$$

is a biholomorphic isomorphism. It follows that the map

$$
\mathfrak{p}^{-} \rightarrow P^{-} Q / Q \subseteq X, \quad z_{1} F_{1}+\cdots+z_{n} F_{n} \mapsto \exp \left(z_{1} F_{1}+\cdots+z_{n} F_{n}\right) Q
$$

is an injective holomorphic map with open image.

\section{Preliminaries from Jordan theory}

Recall that there is a one-to-one correspondence between Hermitian symmetric spaces of compact type and semisimple complex Jordan pairs with positive Hermitian involution. In the following we indicate how to obtain a Jordan pair from the Lie algebra $\mathfrak{g}$. The converse direction is given by the so-called Kantor-Koecher-Tits construction, for which we refer to $\mathrm{Be00}$. Our main reference is Lo75, Lo77, and in particular we use the list of Jordan identities in [Lo77] and refer to single identities by JPxy.

Consider the decomposition $\mathfrak{g}=\mathfrak{p}^{+} \oplus \mathfrak{l} \oplus \mathfrak{p}^{-}$. Then, the Lie bracket defines quadratic operators

$$
Q^{\mp}: \mathfrak{p}^{ \pm} \rightarrow \operatorname{Hom}\left(\mathfrak{p}^{\mp}, \mathfrak{p}^{ \pm}\right), x \mapsto Q_{x}^{ \pm} \quad \text { with } \quad Q_{x}^{\mp}(y):=-\frac{1}{2}[[x, y], x] .
$$

This defines a Jordan pair structure on $\left(\mathfrak{p}^{-}, \mathfrak{p}^{+}\right)$. For convenience, we set $\left(V, V^{\prime}\right):=$ $\left(\mathfrak{p}^{-}, \mathfrak{p}^{+}\right)$, omit the indices \pm on the quadratic operators and define operators via the relations

$$
\{x, y, z\}:=D_{x, y} z:=Q_{x, z} y:=Q_{x+z} y-Q_{x} y-Q_{z} y=-[[x, y], z] .
$$

The context determines the domains of these operators, e.g. $\{,$,$\} is a trilinear map$ from $V \times V^{\prime} \times V$ to $V$ (resp. from $V^{\prime} \times V \times V^{\prime}$ to $V^{\prime}$ ). We also need the Bergman operator $B_{x, y}$ which is defined for all pairs $(x, y) \in V \times V^{\prime}$ by

$$
B_{x, y}:=\operatorname{Id}-D_{x, y}+Q_{x} Q_{y} \in \operatorname{End}(V) .
$$

The pair $(x, y)$ is called quasi-invertible if $B_{x, y}$ is invertible, and then

$$
x^{y}:=B_{x, y}^{-1}\left(x-Q_{x} y\right) \in V
$$

is called the quasi-inverse of $(x, y)$. In the same way one defines the Bergman operator $B_{y, x} \in \operatorname{End}\left(V^{\prime}\right)$ and quasi-inverses $y^{x} \in V^{\prime}$ for pairs $(y, x) \in V^{\prime} \times V$. We note that $B_{x, y}$ is invertible if and only if $B_{y, x}$ is invertible.

The restriction of the Killing form $\kappa: \mathfrak{g} \times \mathfrak{g} \rightarrow \mathbb{C}$ to the product $\mathfrak{p}^{-} \times \mathfrak{p}^{+}$yields a non-degenerate pairing of $V$ and $V^{\prime}$, which is given (up to a constant factor) in Jordan theoretic terms by the trace form,

$$
\tau: V \times V^{\prime} \rightarrow \mathbb{C},(x, y) \mapsto \operatorname{Tr} D_{x, y},
$$

where $\operatorname{Tr}$ denotes the usual trace of linear operators on $V$. This turns $\left(V, V^{\prime}\right)$ into a semisimple complex Jordan pair. 
The Cartan involution $\theta$ of $\mathfrak{g}$ restricted to $\mathfrak{p}^{\mp}$ yields antilinear isomorphisms $V \rightleftarrows V^{\prime}$, which are both denoted by $\bar{x}:=\theta(x)$ and satisfy $Q_{\bar{x}} \bar{y}=\overline{Q_{x} y}$. Moreover, the map

$$
(\mid): V \times V \rightarrow \mathbb{C},(x, z) \mapsto(x \mid z):=\tau(x, \bar{z})
$$

is a positive definite inner product on $V$, and hence $x \mapsto \bar{x}$ is a positive Hermitian involution on $\left(V, V^{\prime}\right)$. By means of this involution, we may identify $V$ with $V^{\prime}$.

3.1. Vector fields and group actions. As in (2), we may identify $V=\mathfrak{p}^{-}$via the exponential map with an open and dense subset of the compact Hermitian symmetric space $X=G / Q$, i.e., $V \hookrightarrow X$ by $x \mapsto \exp (x) Q$. In this way, the automorphism group $G$ acts on $V$ by birational maps, and elements of its Lie algebra $\mathfrak{g}$ can be identified with vector fields on $V$, which turn out to be at most quadratic polynomials. Indeed, according to the decomposition $\mathfrak{g}=\mathfrak{p}^{+} \oplus \mathfrak{l} \oplus \mathfrak{p}^{-}$we have isomorphisms

$$
\begin{aligned}
& \mathfrak{p}^{+} \cong\left\{q_{v}(x):=Q_{x} v \mid v \in V^{\prime}\right\}, \\
& \mathfrak{p}^{-} \cong\{u(x):=u \mid u \in V\},
\end{aligned}
$$

and the vector fields corresponding to $\mathfrak{l}$ are the derivations on $V$, i.e., linear maps $T \in \operatorname{End}(V)$, satisfying

$$
T\{x, y, z\}=\{T x, y, z\}-\left\{x, T^{\#} y, z\right\}+\{x, y, T z\},
$$

for all $x, z \in V, y \in V^{\prime}$, where $T^{\#} \in \operatorname{End}\left(V^{\prime}\right)$ is the adjoint map of $T$ with respect to the trace from $\tau$. As an example, for any pair $(x, y) \in V \times V^{\prime}$, the operator $D_{x, y}$ is a derivation with $\left(D_{x, y}\right)^{\#}=D_{y, x}$. Within the context of Jordan theoretic arguments, we identify the Lie algebra $\mathfrak{g}$ with its realization as vector fields on $V$, so an element $X \in \mathfrak{g}$ is a vector field $\zeta_{X}: V \rightarrow V$ of the form $\zeta_{X}(x)=u+T x+q_{v}(x)$ with $u \in V, T \in \mathfrak{l}$ and $v \in V^{\prime}$. In order to obtain Lie algebra isomorphisms, we note that the commutator of vector fields $\zeta, \eta \in \mathfrak{g}$ is given by

$$
[\zeta, \eta](x)=d \zeta(z) \cdot \eta(z)-d \eta(z) \cdot \zeta(z),
$$

which differs by sign from the usual convention for the Lie bracket of vector fields. In detail, the commutator of two elements $X_{1}=u_{1}+T_{1}+q_{v_{1}}$ and $X_{2}=u_{2}+T_{2}+q_{v_{2}}$ is given by

$$
\left[X_{1}, X_{2}\right]=\left(T_{1} u_{2}-T_{2} u_{1}\right)+\left(D_{u_{2}, v_{1}}+\left[T_{1}, T_{2}\right]-D_{u_{1}, v_{2}}\right)+\left(q_{T_{2}^{\#} v_{1}}-q_{T_{1}^{\#} v_{2}}\right),
$$

and the Killing form $\kappa$ on $\mathfrak{g}$ translates to

$$
\kappa\left(X_{1}, X_{2}\right)=\kappa_{\mathfrak{l}}\left(T_{1}, T_{2}\right)+2 \operatorname{Tr}\left(T_{1} T_{2}\right)-2 \tau\left(u_{1}, v_{2}\right)-2 \tau\left(u_{2}, v_{1}\right),
$$

where $\kappa_{\mathfrak{l}}$ denotes the Killing form on $\mathfrak{l}$. For both formulas; see e.g. [Sa80, §7].

The birational group action of $\exp \left(\mathfrak{p}^{ \pm}\right)$on $V \subseteq X$ is given and denoted by

$$
\begin{array}{ll}
t_{u}(x):=\exp (u) x=x+u & \text { for } \quad u \in V \text { (translation), } \\
\tilde{t}_{v}(x):=\exp \left(q_{v}\right) x=x^{v} & \text { for } \quad v \in V^{\prime} \text { (quasi-translation). }
\end{array}
$$

The subgroup $L \subseteq G$ acts on $V \subseteq X$ by automorphisms of the Jordan pair, i.e. linear transformations linear automorphisms $h \in \mathrm{GL}(V)$ satisfying $h\{x, y, z\}=$ $\left\{h x, h^{-\#} y, h z\right\}$ for all $x, z \in V, y \in V^{\prime}$, where $h^{-\#}:=\left(h^{\#}\right)^{-1}$ and $h^{\#}$ is the adjoint map of $h$ with respect to the trace form $\tau$. In fact, $L$ is a covering group of the identity component of the automorphism group, Aut $\left(V, V^{\prime}\right)$, of the Jordan pair. As an example, for quasi-invertible pairs $(x, y) \in V \times V^{\prime}$, the Bergman operator $B_{x, y}$ is a Jordan pair automorphism with $\left(B_{x, y}\right)^{\#}=B_{y, x}$. 
The Cartan involution $\theta$ corresponding to the compact real forms $\mathfrak{k} \subseteq \mathfrak{l}$ and $\mathfrak{u} \subseteq \mathfrak{g}$ translates to $\theta\left(u+T+q_{v}\right)=\bar{v}-T^{*}+q_{\bar{u}}$, where $T^{*}$ denotes the adjoint of $T$ with respect to the inner product (44) on $V$. Therefore,

$$
\mathfrak{k}=\left\{T \in \mathfrak{l} \mid T=-T^{*}\right\}, \quad \mathfrak{u}=\left\{u+T+q_{\bar{u}} \mid u \in V, T \in \mathfrak{k}\right\} .
$$

In addition, we note that the centre of $\mathfrak{k}$ is given by $\mathfrak{z}(\mathfrak{k})=\mathbb{R}(i \mathrm{Id})$, and the Lie group $K$ is a covering group of the identity component of the group of unitary automorphism, i.e. automorphisms $h \in \operatorname{Aut}\left(V, V^{\prime}\right)$ satisfying $h=h^{-*}$.

3.2. Idempotents, Peirce decomposition, and rank. An idempotent is a pair $\mathbf{e}=\left(e, e^{\prime}\right) \in V \times V^{\prime}$ satisfying the relations $Q_{e} e^{\prime}=e$ and $Q_{e^{\prime}} e=e^{\prime}$. Let $\mathcal{I} \subseteq V \times V^{\prime}$ denote the set of idempotents. For $\mathbf{e} \in \mathcal{I}$, the operators $D_{e, e^{\prime}} \in \operatorname{End}(V)$ and $D_{e^{\prime}, e} \in$ $\operatorname{End}\left(V^{\prime}\right)$ are diagonalizable with spectra in $\{0,1,2\}$, and the decomposition into eigenspaces,

$$
V=V_{2}(\mathbf{e}) \oplus V_{1}(\mathbf{e}) \oplus V_{0}(\mathbf{e}), \quad V^{\prime}=V_{2}^{\prime}(\mathbf{e}) \oplus V_{1}^{\prime}(\mathbf{e}) \oplus V_{0}^{\prime}(\mathbf{e}),
$$

is called the Peirce decomposition with respect to e. We note that in general, $V_{k}^{\prime}(\mathbf{e})$ differs from the image of $V_{k}(\mathbf{e})$ under the involution of $\left(V, V^{\prime}\right)$, i.e., $V_{k}^{\prime}(\mathbf{e}) \neq$ $\overline{V_{k}(\mathbf{e})}$. The Peirce spaces $V_{k}:=V_{k}(\mathbf{e}), V_{k}^{\prime}:=V_{k}^{\prime}(\mathbf{e})$ are subject to the following multiplication rules (the Peirce rules):

$$
\left\{V_{i}, V_{j}^{\prime}, V_{k}\right\} \subseteq V_{i-j+k},\left\{V_{2}, V_{0}^{\prime}, V\right\}=\left\{V_{0}, V_{2}^{\prime}, V\right\}=\{0\},
$$

where $V_{\ell}=\{0\}$ and $V_{\ell}^{\prime}=\{0\}$ if $\ell \notin\{0,1,2\}$. In particular, $\left(V_{k}, V_{k}^{\prime}\right)$ is a subpair of $\left(V, V^{\prime}\right)$. Two idempotents $\mathbf{e}=\left(e, e^{\prime}\right), \mathbf{c}=\left(c, c^{\prime}\right)$ are (strongly) orthogonal if $c \in V_{0}(\mathbf{e})$ or equivalently $e \in V_{0}(\mathbf{c})$. In this case, the sum $\mathbf{e}+\mathbf{c}=\left(e+c, e^{\prime}+c^{\prime}\right)$ is also an idempotent. A non-zero idempotent is called primitive if it is not the sum of two orthogonal non-zero idempotents. A frame of idempotents $\left(\mathbf{e}_{1}, \ldots, \mathbf{e}_{r}\right)$ is a maximal system of primitive orthogonal idempotents. The length $r$ of a frame of idempotents is an invariant of the Jordan pair $\left(V, V^{\prime}\right)$, called the rank and denoted by $\operatorname{rk} V:=r$.

For a system of (pairwise) orthogonal idempotents $\left(\mathbf{e}_{1}, \ldots, \mathbf{e}_{k}\right)$, and so in particular for a frame, the operators $\left(D_{e_{\ell}, e_{\ell}^{\prime}}\right)_{\ell=1, \ldots, k}$ form a commuting set of diagonalizable operators, and hence induce the joint Peirce decomposition

$$
V=\bigoplus_{0 \leq i \leq j \leq k} V_{i j} \quad \text { with } \quad V_{i j}=\left\{x \in V \mid\left\{e_{\ell}, e_{\ell}^{\prime}, x\right\}=\left(\delta_{i \ell}+\delta_{j \ell}\right) x \text { for all } \ell\right\},
$$

and likewise for $\left(D_{e_{\ell}^{\prime}, e_{\ell}}\right)_{\ell=1, \ldots, k}$ and $V^{\prime}$. By definition, $V_{j i}=V_{i j}$ and $V_{j i}^{\prime}=V_{i j}^{\prime}$ for all $i, j$. The Peirce rules refine to the joint Peirce rules

$$
\left\{V_{i j}, V_{j k}^{\prime}, V_{k \ell}\right\} \subseteq V_{i \ell},
$$

and all other types of products vanish. Again, we point out that the image of $V_{i j}$ under the involution of $\left(V, V^{\prime}\right)$ in general differs from $V_{i j}^{\prime}$, unless we consider a special class of idempotents, namely those defined by tripotents, which we discuss in the next section.

If $\left(V, V^{\prime}\right)$ is simple of rank $r$, then, for any primitive idempotent $\mathbf{e} \in \mathcal{I}$, we set

$$
p:=\tau\left(e, e^{\prime}\right)=2+\operatorname{dim} V_{1}(\mathbf{e}),
$$

which is independent of the choice of $\mathbf{e}$. This structure constant of $\left(V, V^{\prime}\right)$ appears in subsequent formulas which involve the Killing form of $\mathfrak{g}$.

We also need the notion of rank for arbitrary elements $x \in V$ or $y \in V^{\prime}$. For $x \in V$ the subspace $[x]:=Q_{x} V^{\prime} \subseteq V$ is called the principal inner ideal generated by $x$. The 
rank of $x, \operatorname{rk} x$, is defined as the maximum length of all chains $\left[x_{0}\right] \nsubseteq\left[x_{1}\right] \mp \cdots \mp\left[x_{k}\right]$ with $x_{i} \in[x]$. Similarly, one defines $[y]:=Q_{y} V \subseteq V^{\prime}$ and $\operatorname{rk} y$ for $y \in V^{\prime}$. If $\mathbf{e}=\left(e, e^{\prime}\right)$ is an idempotent, then the Peirce rules imply $[e]=V_{2}(\mathbf{e})$ and $\left[e^{\prime}\right]=V_{2}^{\prime}(\mathbf{e})$, and it turns out $([\mathrm{Lo91}, \S 3])$ that $\mathrm{rk} e=\mathrm{rk} e^{\prime}$. We therefore define rke $\mathbf{e}=\mathrm{rk} e$ and call this the rank of the idempotent $\mathbf{e}$. The set $\mathcal{I}$ of idempotents therefore decomposes into subsets of constant rank idempotents, denoted by $\mathcal{I}_{k}:=\{\mathbf{e} \in \mathcal{I} \mid \operatorname{rk} \mathbf{e}=k\}$. Since we assume $\left(V, V^{\prime}\right)$ to be finite dimensional and simple, each element $e \in V$ admits a completion to an idempotent, i.e., an element $e^{\prime} \in V^{\prime}$ such that $\left(e, e^{\prime}\right) \in \mathcal{I}$. From this, it follows that the decomposition of an idempotent $\mathbf{e}$ of rank $k$ into primitive orthogonal idempotents has exactly $k$ summands. Therefore, the maximum of all ranks of elements in $V$ coincides with the rank of $V$ as it is defined above.

3.3. Tripotents and spectral decomposition. The involution on $\left(V, V^{\prime}\right)$ admits the definition of (odd) powers of elements, namely for $x \in V$ define $x^{(1)}:=x$ and inductively $x^{(2 k+1)}:=Q_{x} \overline{x^{(2 k-1)}}$ for $k \geq 1$. An element $e \in V$ is called a tripotent if $e^{(3)}=e$, i.e., $e=Q_{e} \bar{e}$. Equivalently, $e$ is a tripotent if and only if $(e, \bar{e})$ is an idempotent. In particular, all notions defined in the last section apply to the idempotent $(e, \bar{e})$. Without causing ambiguities in notation, we may identify $e$ with $(e, \bar{e})$ if necessary. Concerning the Peirce decomposition, we note that $V_{k}^{\prime}(e)=\overline{V_{k}(e)}$ for a tripotent $e$, and $V_{i j}^{\prime}=\overline{V_{i j}}$ for a system of orthogonal tripotents $\left(e_{1}, \ldots, e_{k}\right)$. The set of tripotents is denoted by $S \subseteq V$.

For the explicit description of the moment map on $X$, we will make use of the following spectral theorem [Lo77, §3.12].

Theorem 3.1 (Spectral decomposition). Let $\left(V, V^{\prime}\right)$ be a finite dimensional semisimple Jordan pair with positive Hermitian involution. Then every element $x \in V$ admits a unique decomposition

$$
x=\sigma_{1} e_{1}+\cdots+\sigma_{k} e_{k}, \quad \sigma_{1}>\cdots>\sigma_{k}>0,
$$

where the $e_{i}$ are pairwise orthogonal non-zero tripotents which are real linear combinations of powers of $x$, and $\sigma_{i} \in \mathbb{R}$.

3.4. Idempotents and roots. Idempotents are related to $\mathfrak{s l}_{2}$-triples in $\mathfrak{g}$ in the following way: If $\left(e, e^{\prime}\right) \in \mathcal{I}$ is an idempotent, then $(E, H, F):=\left(-q_{e^{\prime}},-D_{e, e^{\prime}}, e\right)$ is an $\mathfrak{s l}_{2}$-triple in $\mathfrak{g}$ with $-q_{e^{\prime}} \in \mathfrak{p}^{+},-D_{e, e^{\prime}} \in \mathfrak{l}$ and $e \in \mathfrak{p}^{-}$(a constant vector field). Indeed, according to (6), it follows that

$$
\begin{aligned}
& {\left[-D_{e, e^{\prime}},-q_{e^{\prime}}\right]=-q_{D_{e^{\prime}, e} e^{\prime}}=2\left(-q_{e^{\prime}}\right),} \\
& {\left[-D_{e, e^{\prime}}, e\right]=-D_{e, e^{\prime}} e=-2 e,} \\
& {\left[-q_{e^{\prime}}, e\right]=-D_{e, e^{\prime}} .}
\end{aligned}
$$

Conversely, if $(E, H, F)$ is an $\mathfrak{s l}_{2}$-triple in $\mathfrak{g}$ with $E \in \mathfrak{p}^{+}, H \in \mathfrak{l}$ and $F \in \mathfrak{p}^{-}$, then the identifications $V^{\prime} \cong \mathfrak{p}^{+}$and $V \cong \mathfrak{p}^{-}$in (5) yield an idempotent $\left(e, e^{\prime}\right) \in \mathcal{I}$ with $E=-q_{e^{\prime}}, F=e$ and $H=-D_{e, e^{\prime}}$.

This correspondence between idempotents and certain $\mathfrak{s l}_{2}$-triples in $\mathfrak{g}$ also applies to tripotents: Starting with a tripotent $e \in S$, we obtain the $\mathfrak{s l}_{2}$-triple $(E, H, F):=$ $\left(-q_{\bar{e}},-D_{e, \bar{e}}, e\right)$ with the additional property $\theta(E)=-F$, where $\theta$ denotes the Cartan involution on $\mathfrak{g}$, and hence $i H \in \mathfrak{k}$. Conversely, any $\mathfrak{s l}_{2}$-triple $(E, H, F)$ with $E \in \mathfrak{p}^{+}$ and $\theta(E)=-F$ yields a tripotent $e \in S$ corresponding to $E=-q_{\bar{e}}$. 
In particular, the $\mathfrak{s l}_{2}$-triples associated to the system of strongly orthogonal roots $\gamma_{1}, \ldots, \gamma_{r}$ yield a system $\left(e_{1}, \ldots, e_{r}\right)$ of tripotents, and it is straightforward to see that strong orthogonality of the roots is equivalent to strong orthogonality of the tripotents. Therefore, $\left(e_{1}, \ldots, e_{r}\right)$ is a frame associated to the system of strongly orthogonal roots. We summarize the situation by

$$
\left(E_{\gamma_{j}}, H_{\gamma_{j}}, F_{\gamma_{j}}\right)=\left(-q_{\bar{e}_{j}},-D_{e_{j}, \bar{e}_{j}}, e_{j}\right) .
$$

Moreover, using the Killing form (7), the relation $\gamma_{j}=c \cdot \kappa\left(H_{\gamma_{j}},-\right)$ with $c=$ $2 / \kappa\left(H_{\gamma_{j}}, H_{\gamma_{j}}\right)$ yields

$$
\gamma_{j}(T)=-\frac{1}{p} \tau\left(T e_{j}, \bar{e}_{j}\right) \text { for all } T \in \mathfrak{l},
$$

where $p$ is the structure constant defined by (8). This is the Jordan theoretic description of the strongly orthogonal roots.

Recall the decomposition (11) of the maximal abelian subalgebra $\mathfrak{t}$ of $\mathfrak{k}$ into $\mathfrak{t}=$ $\mathfrak{s} \oplus \mathfrak{s}^{\perp}$. In Jordan theoretic terms, we obtain

$$
\mathfrak{s}=\left\langle i D_{e_{j}, \bar{e}_{j}}, i \operatorname{Id}_{V} \mid j=1, \ldots, r\right\rangle_{\mathbb{R}},
$$

and $\mathfrak{s}^{\perp} \subseteq\left\{T \in \mathfrak{k} \mid T e_{j}=0\right.$ for all $\left.\mathrm{j}\right\}$.

3.5. Determinants. We have to deal with two kinds of determinants. On the one hand, there is the Jordan pair determinant $\Delta: V \times V^{\prime} \rightarrow \mathbb{C}$ associated to a Jordan pair $\left(V, V^{\prime}\right)$, often also called the generic minimum polynomial; cf. [Lo75, §16]. On the other hand, let $\mathbf{e}=\left(e, e^{\prime}\right)$ be an idempotent in $\left(V, V^{\prime}\right)$. Then the Peirce 2-space $V_{2}(e)$ becomes a unital Jordan algebra with product $x \circ z:=\frac{1}{2}\left\{x, e^{\prime}, z\right\}$ and unit element $e$. The Jordan algebra determinant corresponding to $V_{2}(e)$ is denoted by $\Delta_{\mathbf{e}}: V_{2}(\mathbf{e}) \rightarrow \mathbb{C}$. Likewise, $V_{2}^{\prime}(\mathbf{e})$ is a Jordan algebra with product $y \circ w:=$ $\frac{1}{2}\{y, e, w\}$, unit element $e^{\prime}$, and Jordan algebra determinant $\Delta_{\mathbf{e}}^{\prime}: V_{2}^{\prime}(\mathbf{e}) \rightarrow \mathbb{C}$. The connection between the Jordan pair determinant and Jordan algebra determinants is given as follows:

Lemma 3.2. If $\mathbf{e}=\left(e, e^{\prime}\right)$ is an idempotent, then

$$
\Delta_{\mathbf{e}}(x)=\Delta\left(e-x, e^{\prime}\right), \quad \Delta_{\mathbf{e}}^{\prime}(y)=\Delta\left(e, e^{\prime}-y\right)
$$

for all $x \in V_{2}(\mathbf{e})$ and $y \in V_{2}^{\prime}(\mathbf{e})$.

Proof. Set $A:=V_{2}(\mathbf{e})$ and $A^{\prime}:=V_{2}^{\prime}(\mathbf{e})$. Due to [Lo75, §3.4], the maps $Q_{e}: A^{\prime} \rightarrow A$ and $Q_{e^{\prime}}: A \rightarrow A^{\prime}$ are homomorphisms of Jordan algebras, and since $Q_{e} Q_{e^{\prime}}=\operatorname{Id}_{A}$ and $Q_{e^{\prime}} Q_{e}=\operatorname{Id}_{A^{\prime}}$, these are in fact isomorphisms. Therefore, $\Delta_{\mathbf{e}}^{\prime}(y)=\Delta_{\mathbf{e}}\left(Q_{e} y\right)$ for all $y \in A^{\prime}$. The determinant of the Jordan subpair $\left(A, A^{\prime}\right)$ is just the restriction of the Jordan pair determinant, $\Delta$, to $A \times A^{\prime}$. Due to [Lo75, §16.3(ii)], this yields the relation

$$
\Delta\left(e, e^{\prime}-y\right)=\Delta_{\mathbf{e}}(e) \cdot \Delta_{\mathbf{e}}\left(Q_{e} y\right)=\Delta_{\mathbf{e}}^{\prime}(y)
$$

for all $y \in A^{\prime}$. Now, $\Delta_{\mathbf{e}}(x)=\Delta\left(e-x, e^{\prime}\right)$ is a consequence of the identity $\Delta\left(x, Q_{y} z\right)=$ $\Delta\left(z, Q_{y} x\right)$; see [Lo75, §3.5].

In the following, we use (10) to extend the Jordan algebra determinants $\Delta_{\mathbf{e}}$ and $\Delta_{\mathrm{e}}^{\prime}$ to polynomial maps on $V$ and $V^{\prime}$. By abuse of notation, these extensions are also denoted by $\Delta_{\mathbf{e}}$ and $\Delta_{\mathbf{e}}^{\prime}$. We note that if $V=V_{2}(\mathbf{e}) \oplus V_{1}(\mathbf{e}) \oplus V_{0}(\mathbf{e})$ is the Peirce decomposition with respect to $\mathbf{e}$, then $\Delta_{\mathbf{e}}$ vanishes on $V_{1}(\mathbf{e}) \oplus V_{0}(\mathbf{e})$. Analogous 
results hold for $\Delta_{\mathbf{e}}^{\prime}$. For later use, we note the following relation between the rank of idempotents and zeros of Jordan algebra determinants:

Lemma 3.3. Let $x \in V$ be a fixed element and $k \in \mathbb{N}$. Then, $\Delta_{\mathbf{c}}(x)=0$ for all idempotents $\mathbf{c}$ of rank $k$ if and only if $k>\operatorname{rk} x$. The same holds for $y \in V^{\prime}$ and $\Delta_{\mathbf{c}}^{\prime}(y)=0$ in place of $\Delta_{\mathbf{c}}(x)=0$.

Proof. First assume that $k \leq \operatorname{rk} x$. Let $\mathbf{e}=\left(e, e^{\prime}\right)$ be a completion of $e=x$ to an idempotent, and let $\mathbf{e}=\mathbf{e}_{1}+\cdots+\mathbf{e}_{\ell}$ be a decomposition into primitive orthogonal idempotents. Then, $\ell=\operatorname{rk} \mathbf{e}=\operatorname{rk} x \geq k$, and hence $\mathbf{c}:=\mathbf{e}_{1}+\cdots+\mathbf{e}_{k}$ is well defined and satisfies $\Delta_{\mathbf{c}}(x)=\Delta_{\mathbf{c}}(e)=1$. This proves the 'only if' part. For the converse direction assume $\Delta_{\mathbf{c}}(x) \neq 0$ for some $\mathbf{c} \in \mathcal{I}_{k}$. Let $x=x_{2}+x_{1}+x_{0}$ be the components of $x$ in the Peirce decomposition of $V$ with respect to $\mathbf{c}$. Then, $\Delta_{\mathbf{c}}(x)=\Delta_{\mathbf{c}}\left(x_{2}\right)$, and this is non-vanishing if and only if $x_{2}$ is invertible in the unital Jordan algebra $V_{2}(\mathbf{c})$. Furthermore, this is equivalent to the identity $\left[x_{2}\right]=V_{2}(\mathbf{c})$, where $\left[x_{2}\right]=Q_{x_{2}} V^{\prime}$ is the principal inner ideal corresponding to $x_{2}$. Since $V_{2}(\mathbf{c})=[c]$, this implies that $\operatorname{rk} x_{2}=\mathrm{rk} c=\mathrm{rk} \mathbf{c}$. Now the statement follows from the inequality $\operatorname{rk} x_{2} \leq \mathrm{rk} x$; $\mathrm{cf}$. [Lo91, §3].

3.6. Jordan theoretic model of $X$. The concept of quasi-inverses provides a Jordan theoretic model for the compact Hermitian symmetric space $X=G / Q$ (due to O. Loos [Lo77]): For each $a \in V^{\prime}$ the map $\iota_{a}: V \rightarrow X$ given by $\iota_{a}(x)=$ $\exp \left(q_{a}\right) \exp (x) Q=\tilde{t}_{a} t_{x} Q$ is an open and dense imbedding of $V$ in $X$. This yields an open covering of $X$ by subsets $X_{a}:=\iota_{a}(V), a \in V^{\prime}$. It turns out that $X_{a} \cap X_{b}$ is the image of $\{x \in V \mid(x, a-b)$ quasi-invertible $\}$ under $\iota_{a}$, and the transition map $\varphi_{b}^{a}=\iota_{b}^{-1} \circ \iota_{a}$ is given by

$$
\varphi_{b}^{a}(x)=x^{a-b} .
$$

This description of $X$ may be summarized in the equivalence relation $X \cong(V \times$ $\left.V^{\prime}\right) / R$ with

$$
(x, a) R(\tilde{x}, b) \Longleftrightarrow\left\{\begin{array}{l}
(x, a-b) \text { is quasi-invertible } \\
\text { and } \tilde{x}=x^{a-b} .
\end{array}\right.
$$

The equivalence class of an element $(x, a)$ is denoted by $[x: a]$. For $a=0$, the imbedding $\iota_{0}$ is just the standard imbedding of $V \cong \mathfrak{p}^{-}$into $X$, which we have already used in Section 3.1. In the following, we write $V \subseteq X$ for the identification of $V$ with its image under $\iota_{0}$ in $X$.

Some questions require yet another description of the elements of $X$. Consider the description via equivalence classes as it is given in (11). Whereas the chart maps concern elements with fixed second entry $a \in V^{\prime}$, i.e., $x \mapsto[x: a]$, the following proposition selects for each element in $X$ a representative which is adapted to arguments concerning the action of the automorphism group of $\left(V, V^{\prime}\right)$ on $X$.

Proposition 3.4. Let $[x: a]$ be an element of $X=\left(V \times V^{\prime}\right) / R$. Then, there exist an idempotent $\mathbf{e}=\left(e, e^{\prime}\right) \in \mathcal{I}$ and an element $z \in V_{0}(\mathbf{e})$ such that $[x: a]=\left[e+z: e^{\prime}\right]$. Moreover, the idempotent $\mathbf{e}$ can be chosen to be tripotent, i.e., $\mathbf{e}=(e, \bar{e})$.

Proof. The existence of a representative for $[x: a]$ of the form $\left(e+z, e^{\prime}\right)$ with idempotent $\mathbf{e}$ and $z \in V_{0}(\mathbf{e})$ is proved in [Lo94]; see Theorem 3.8, Proposition 4.6, and Theorem 4.7 therein. The possibility of choosing e to be tripotent follows from the fact that in our setting $\left(\left(V, V^{\prime}\right)\right.$ being finite dimensional and simple), for any 
idempotent e there exists a tripotent $c$ such that $V_{2}(\mathbf{e})=V_{2}(c)$. Therefore, we may assume that the idempotent $e=\left(e_{+}, e_{-}\right)$used in the last part of Proposition 6.5 in Lo94] is in fact a tripotent.

\section{Line BUNDLES AND REPRESENTATION SPACES FOR $G$}

4.1. Prequantum bundles on $G / Q$. Let $-\lambda \in \mathfrak{h}^{*}$ be the fundamental weight associated to the simple root $\beta_{1}$. Let $\chi_{\lambda}: Q \rightarrow \mathbb{C}^{\times}$be the holomorphic character determined by the condition $\left.d \chi_{\lambda}(e)\right|_{\mathfrak{h}}=\lambda$, and let $\mathscr{L}:=G \times_{Q} \mathbb{C} \rightarrow X$ be the line bundle associated to $\chi_{\lambda}$. We shall be concerned with the family $H^{0}\left(X, \mathscr{L}^{k}\right)$, for $k \in \mathbb{N}$, of $G$-representations, and with the decomposition under the group $L$. Notice that $K$ is a maximal compact subgroup of $L$ so that the decompositions under $L$ amount to the decompositions under $K$.

In order to realize the homogeneous space $X$ as a coadjoint $U$-orbit in $\mathfrak{u}^{*}$, we extend the $\mathbb{C}$-linear functional $\lambda \in \mathfrak{h}^{*}$ to a functional on $\mathfrak{g}$ by requiring that it annihilates all root spaces $\mathfrak{g}_{\alpha}$. This convention will hereafter be used for extending linear functionals on $\mathfrak{h}$ to linear functionals on $\mathfrak{g}$. Similarly, we extend $\mathbb{R}$-linear functionals on $\mathfrak{t}$ to $\mathbb{R}$-linear functionals on $\mathfrak{u}$.

Notice that $\lambda$ has imaginary values on $\mathfrak{t}$ so that $i \lambda$ restricts to a real-valued $\mathbb{R}$-linear functional on $\mathfrak{t}$. We will write $i \lambda$ instead of $\left.i \lambda\right|_{\mathfrak{u}}$ for the induced $\mathbb{R}$-linear functional on $\mathfrak{u}$. Using the above conventions, $X$ can be realized as the coadjoint orbit, $\mathcal{O}_{\lambda} \subseteq \mathfrak{u}^{*}$, of $i \lambda$. When $\mathcal{O}_{\lambda}$ is equipped with the Kostant-Kirillov symplectic form, $\omega_{\lambda}$, the action of $U$ is Hamiltonian with moment map

$$
\mu: \mathcal{O}_{\lambda} \rightarrow \mathfrak{u}^{*}
$$

being the inclusion $\mathcal{O}_{\lambda} \subseteq \mathfrak{u}^{*}$. The action of the subgroup $K$ is also Hamiltonian, with moment map

$$
\mu_{\mathfrak{k}}: \mathcal{O}_{\lambda} \rightarrow \mathfrak{k}^{*}, \quad \mu_{\mathfrak{k}}(f)(x):=f(x), \quad f \in \mathcal{O}_{\lambda}, \quad x \in \mathfrak{k} .
$$

The line bundle $\mathscr{L}$ is a prequantum line bundle for the Kostant-Kirillov form on $\mathcal{O}_{\lambda}$. In order to interpret the tensor powers $\mathscr{L}^{k}$ as prequantum line bundles on $X \cong \mathcal{O}_{\lambda}$, we use the natural isomorphism $\mathcal{O}_{\lambda} \cong \mathcal{O}_{k \lambda}, f \mapsto k f$, to identify the symplectic manifolds $\left(\mathcal{O}_{k \lambda}, \omega_{k \lambda}\right)$ and $\left(\mathcal{O}_{\lambda}, k \omega_{\lambda}\right)$. Then $\mathscr{L}^{k}$ is a prequantum line bundle for $\left(\mathcal{O}_{\lambda}, k \omega_{\lambda}\right)$. The moment map for the $K$-action on $\left(\mathcal{O}_{\lambda}, k \omega_{\lambda}\right)$ is

$$
\mu_{\mathfrak{k}}^{k}:=k \mu_{\mathfrak{k}} .
$$

4.2. Trivialization of sections. Consider the dual space $H^{0}(X, \mathscr{L})^{*}$, which is a highest weight module of highest weight $-\lambda$. We make a specific choice of a highest weight vector. For this purpose we identify the space of holomorphic sections $H^{0}(X, \mathscr{L})$ with the space of $Q$-equivariant holomorphic functions $F: G \rightarrow \mathbb{C}$ having the $Q$-equivariance property

$$
F(g q)=\chi_{\lambda}^{-1}(q) F(g), \quad g \in G, \quad q \in Q .
$$

Define $w_{1} \in H^{0}(X, \mathscr{L})^{*}$ as the linear functional $F \mapsto \mathrm{ev}_{e}(F):=F(e)$. Then $w_{1}$ is a cyclic vector for $H^{0}(X, \mathscr{L})^{*}$ as a $U(\mathfrak{g})$-module, as well as a $U\left(\mathfrak{p}^{-}\right)$-module. For $k \in \mathbb{N}$, the vector $w_{k}:=w_{1}^{\otimes k} \in H^{0}\left(X, \mathscr{L}^{k}\right)^{*}$ is then a highest weight vector for $H^{0}\left(X, \mathscr{L}^{k}\right)^{*}$. Given these normalizations of highest weight vectors of the spaces $H^{0}\left(X, \mathscr{L}^{k}\right)^{*}$, we now consider local trivializations of the bundles $\mathscr{L}^{k}$.

The principal bundle $G \rightarrow G / Q$ is trivial over the open set $P^{-} Q / Q$. Hence the bundles $\mathscr{L}^{k}$, being associated to this principal bundle, are also trivial over 
$P^{-} Q / Q$. If we identify a section $\varphi$ of $\mathscr{L}^{k}$ with a linear functional on the dual space $H^{0}\left(X, \mathscr{L}^{k}\right)^{*}$, then the realization of $\varphi$ as a $Q$-equivariant holomorphic function, $F$, on $G$ is given by

$$
F(g)=\varphi\left(g \cdot w_{k}\right)
$$

The trivialization of a section, viewed as a $Q$-equivariant holomorphic function $F: G \rightarrow \mathbb{C}$, is given by the restriction of $F$ to $P^{-}$. In the local coordinates (2), the restriction of $F$ to $P^{-}$is given by the function

$$
f\left(z_{1}, \ldots, z_{n}\right)=\varphi\left(\exp \left(z_{1} F_{1}+\cdots+z_{n} F_{n}\right) \cdot w_{k}\right), \quad z \in \mathfrak{p}^{-} .
$$

Proposition 4.1. Let $s \in H^{0}\left(X, \mathscr{L}^{k}\right)$ be a weight vector of weight $\delta$. If scalar multiples of the two monomial terms $z^{a}$ and $z^{b}$ occur in the polynomial that trivializes $s$, then the identity

$$
k \lambda+\sum_{j=1}^{n} a_{j} \alpha_{j}=k \lambda+\sum_{j=1}^{n} b_{j} \alpha_{j}
$$

holds in the weight lattice. Moreover, $\delta=k \lambda+\sum_{j=1}^{n} a_{j} \alpha_{j}$.

Proof. The holomorphic section $s$ corresponds to a linear functional $\varphi$ on the space $H^{0}\left(X, \mathscr{L}^{k}\right)^{*}$. The local trivialization of $s$ is then given by the polynomial function

$$
\begin{aligned}
f\left(z_{1}, \ldots, z_{n}\right) & =\varphi\left(\exp \left(z_{1} F_{1}+\cdots+z_{n} F_{n}\right) \cdot w_{k}\right) \\
& =\sum_{c \in \mathbb{N}_{0}^{n}} \frac{1}{\left(c_{1} !\right) \cdots\left(c_{n} !\right)} z^{c} \varphi\left(F^{c} \cdot w_{k}\right),
\end{aligned}
$$

where $F^{c}:=F_{1}^{c_{1}} \cdots F_{n}^{c_{n}}$. Since each vector $F^{c} \cdot w_{k}$ is a weight vector (of weight $-k \lambda-\sum_{j=1}^{n} c_{j} \alpha_{j}$ ), the functional $\varphi$ can only have non-zero values on vectors $F^{c} . w_{k}$ of the fixed weight $-\delta$.

We now define a valuation-like function

$$
v: \bigsqcup_{k \in \mathbb{N}} H^{0}\left(X, \mathscr{L}^{k}\right) \backslash\{0\} \rightarrow \mathbb{N}_{0}^{n},
$$

i.e., a function satisfying the properties

$$
\begin{aligned}
& v(s t)=v(s)+v(t), \quad s \in H^{0}\left(X, \mathscr{L}^{k}\right) \backslash\{0\}, t \in H^{0}\left(X, \mathscr{L}^{\ell}\right) \backslash\{0\}, \\
& v(s+t) \geq \min (v(s), v(t)), \quad s, t \in H^{0}\left(X, \mathscr{L}^{k}\right) \backslash\{0\}, s+t \neq 0, \\
& v(\lambda s)=v(s), \quad s \in H^{0}\left(X, \mathscr{L}^{k}\right) \backslash\{0\}, \lambda \in \mathbb{C}^{\times} .
\end{aligned}
$$

If $s \in H^{0}\left(X, \mathscr{L}^{k}\right) \backslash\{0\}$ is locally trivialized as the polynomial $f=\sum_{a \in \mathbb{N}_{0}^{n}} c_{a} z^{a}$, let

$$
v(s):=\min \left\{a \in \mathbb{N}_{0}^{n} \mid c_{a} \neq 0\right\},
$$

where the minimum refers to the inverse lexicographic order on $\mathbb{Z}^{n}$.

Remark 4.2. The function $v$, although it seems to depend on the particular local coordinates chosen, has a global geometric meaning. In fact, $v$ can be interpreted as defining the "successive orders of vanishing" of $s$ along a flag of irreducible subvarieties $X_{0} \subseteq \ldots \subseteq X_{n-1} \subseteq X_{n}=X$, where $\operatorname{dim} X_{i}=i$, in the setting defined by Okounkov ([0k96]) and later developed by Lazarsfeld-Mustaţă ([LM09]). This 
interpretation will however not play any role in the rest of this paper. Instead, the particular local expression (14) will be useful.

To the function $v$ we attach the semigroup

$$
S\left(\mathscr{L}, N_{L}^{+}, v\right):=\left\{(k, v(s)) \mid s \in H^{0}\left(X, \mathscr{L}^{k}\right)^{N_{L}^{+}} \backslash\{0\}\right\} \subseteq \mathbb{N} \times \mathbb{N}_{0}^{n}
$$

and the closed convex cone, $C\left(\mathscr{L}, N_{L}^{+}, v\right)$, in $\mathbb{R} \times \mathbb{R}^{n}$, which is generated by the semigroup $S\left(\mathscr{L}, N_{L}^{+}, v\right)$. Finally, we define the Okounkov body,

$$
\Delta\left(\mathscr{L}, N_{L}^{+}, v\right):=C\left(\mathscr{L}, N_{L}^{+}, v\right) \cap\left(\{1\} \times \mathbb{R}^{n}\right) .
$$

\section{5. $L$-TYPES AND $L$-ORBITS IN $X$}

We give a geometric proof of the decomposition of $H^{0}(X, \mathscr{L})$ into irreducible $L$ modules. More precisely, we claim that the decomposition is obtained by restriction to the closed $L$-orbits in $X=G / Q$. Recall that $L$ is a covering group of the identity component of the automorphism group of the Jordan pair $\left(V, V^{\prime}\right)$.

5.1. Closed $L$-orbits. Recall that the set $\mathcal{I} \subseteq V \times V^{\prime}$ of idempotents decomposes into the subsets of constant rank idempotents,

$$
\mathcal{I}=\biguplus_{k=0}^{r} \mathcal{I}_{k} \quad \text { with } \quad \mathcal{I}_{k}=\{\mathbf{e} \in \mathcal{I} \mid \mathrm{rk} \mathbf{e}=k\},
$$

where $r$ denotes the rank of the Jordan pair $\left(V, V^{\prime}\right)$. Assuming that $\left(V, V^{\prime}\right)$ is simple (or equivalently, that the Hermitian symmetric space $X$ is irreducible), it is well known that the $\mathcal{I}_{k}$ are the connected components of $\mathcal{I}$ (with respect to the induced topology from $V \times V^{\prime}$ ) and that the $L$-action on $V \times V^{\prime}$ restricts to a transitive $L$-action on each component $\mathcal{I}_{k} \subseteq V \times V^{\prime}$; cf. [Lo75, §17.1]. Let $N_{k}$ be the dimension of the Peirce 2-space $\operatorname{dim} V_{2}(\mathbf{e})$ for some idempotent $\mathbf{e} \in \mathcal{I}_{k}$, which is independent of the choice of $\mathbf{e}$, and let $\mathrm{Gr}_{N_{k}}(V)$ be the (classical) Grassmannian manifold of $N_{k}$-dimensional subspaces in $V$, equipped with the natural $L$-action induced by the $L$-action on $V$. Consider the subset

$$
\mathcal{P}_{k}:=\left\{U \subseteq V \mid U=V_{2}(\mathbf{e}) \text { for some } \mathbf{e} \in \mathcal{I}_{k}\right\} \subseteq \operatorname{Gr}_{N_{k}}(V) .
$$

Since $V_{2}(h \mathbf{e})=h V_{2}(\mathbf{e})$ for any $h \in L$ and $\mathbf{e} \in \mathcal{I}_{k}$, the map

$$
\pi_{k}: \mathcal{I}_{k} \rightarrow \mathcal{P}_{k}, \mathbf{e} \mapsto V_{2}(\mathbf{e})
$$

is $L$-equivariant, and hence $\mathcal{P}_{k}$ is an $L$-orbit in $\operatorname{Gr}_{N_{k}}(V)$. The next lemma shows that the $L$-stabilizer of some element in $\mathcal{P}_{k}$ is a parabolic subgroup. Therefore $\mathcal{P}_{k}$ is a closed $L$-orbit and hence a smooth projective variety called the Peirce variety of rank $k$.

Lemma 5.1. Let $\left(e_{1}, \ldots, e_{r}\right)$ denote the frame of tripotents corresponding to the Cartan subalgebra $\mathfrak{h}$ of $\mathfrak{l}$ as it is defined in Section 2, For $k=0, \ldots, r$ let $\epsilon_{k}:=\sum_{i=1}^{k} e_{i}$. Then, the stabilizer subgroup $Q_{k} \subseteq L$ of the Peirce 2-space $V_{2}\left(\epsilon_{k}\right)$ contains the opposite Borel subgroup $B_{L}^{\mathrm{op}}$.

Proof. This proof is based on the ideas of [Up86]. Since the Peirce variety $\mathcal{P}_{k}$ is a projective variety, the stabilizer subgroup $Q_{k} \subseteq L$ is parabolic, and hence it suffices to prove this lemma on the Lie algebra level, i.e., we show that $\mathfrak{b}_{L}^{\text {op }} \subseteq \mathfrak{q}_{k}$ with $\mathfrak{q}_{k}=$ $\left\{T \in \mathfrak{l} \mid T V_{2}\left(\epsilon_{k}\right) \subseteq V_{2}\left(\epsilon_{k}\right)\right\}$. Let $V=\oplus_{0 \leq i \leq j \leq r} V_{i j}$ be the joint Peirce decomposition 
with respect to the frame of tripotents $\left(e_{1}, \ldots, e_{r}\right)$, and define

$$
\left.\mathfrak{l}_{i j}:=\left\langle D_{u, \bar{v}} \in \mathfrak{l}\right| u \in V_{i \ell}, v \in V_{\ell j} \text { for some } \ell\right\rangle_{\mathbb{C}},
$$

where $\langle\cdots\rangle_{\mathbb{C}}$ denotes the $\mathbb{C}$-linear span. We note that $\mathfrak{l}_{i j} \neq \mathfrak{l}_{j i}$ for $i \neq j$. We claim that

$$
\mathfrak{l}=\mathfrak{l}_{0} \oplus \bigoplus_{i \neq j} \mathfrak{l}_{i j} \quad \text { with } \quad \mathfrak{l}_{0}:=\bigoplus_{i=1}^{r} \mathfrak{l}_{i i}
$$

is the weight space decomposition of $\mathfrak{l}$ with respect to the adjoint action of the abelian subalgebra $\mathfrak{s}=\left\langle i D_{e_{j}, \bar{e}_{j}}, i \operatorname{Id}_{V} \mid j=1, \ldots, r\right\rangle_{\mathbb{R}} \subseteq \mathfrak{t}$. Indeed, since $\mathfrak{l}=\left[\mathfrak{p}^{+}, \mathfrak{p}^{-}\right]$, $\mathfrak{l}$ is generated by all derivations of the form $D_{u, \bar{v}}$ with $u, v \in V$, and for $D_{u, \bar{v}} \in \mathfrak{l}_{i j}$, the Jordan identity JP15 implies

$$
\left[D_{e_{k}, \bar{e}_{k}}, D_{u, \bar{v}}\right]=D_{\left\{e_{k}, \bar{e}_{k}, u\right\}, \bar{v}}-D_{u,\left\{\bar{e}_{k}, e_{k}, \bar{v}\right\}}=\left(\delta_{k i}-\delta_{k j}\right) D_{u, \bar{v}},
$$

where $\delta_{i j}$ is Kronecker's delta. Moreover, $i \operatorname{Id}_{V}$ acts trivially on each $\mathfrak{l}_{i j}$. With (9), it readily follows that the weight corresponding to $\mathfrak{l}_{i j}$ is $\left(\gamma_{i}-\gamma_{j}\right) / 2$, where we also set $\gamma_{0}:=0$.

Now let $\mathfrak{l}=\mathfrak{t} \oplus \oplus_{\alpha \in \Phi_{L}} \mathfrak{l}_{\alpha}$ be the root decomposition of $\mathfrak{l}$ with respect to the maximal torus $\mathfrak{t}$. By [HC56, Lemma 13], a positive root $\alpha \in \Phi_{L}^{+}$either vanishes on $\mathfrak{s}$ or its restriction to $\mathfrak{s}$ is of the form $\left(\gamma_{i}-\gamma_{j}\right) / 2$ with $1 \leq j<i \leq r$ or $-\gamma_{i} / 2$. We thus obtain that

$$
\mathfrak{p}:=\bigoplus_{1 \leq i \leq j \leq r} \mathfrak{l}_{i j} \oplus \bigoplus_{i=1}^{r} \mathfrak{l}_{i 0}
$$

is a subalgebra of $\mathfrak{l}$ which contains the opposite Borel subalgebra $\mathfrak{b}_{L}^{\text {op }}$.

In the last step of the proof, we show that $\mathfrak{p} \subseteq \mathfrak{q}_{k}$. Due to the derivation property of elements $T \in \mathfrak{p}$, the relation $T \epsilon_{k} \in V_{2}\left(\epsilon_{k}\right)$ already implies $T V_{2}\left(\epsilon_{k}\right) \subseteq V_{2}\left(\epsilon_{k}\right)$. It suffices to verify this relation for the linear generators $T=D_{u, \bar{v}} \in \mathfrak{l}_{i j}$ with $u \in V_{i \ell}$, $v \in V_{\ell j}$ for some $\ell$. First assume that $T \in \mathfrak{l}_{i i}$. Since $V_{i \ell}$ is contained in one of the Peirce spaces $V_{m}\left(\epsilon_{k}\right)$ with $m \in\{0,1,2\}$, the Peirce rules imply $D_{u, \bar{v}} \epsilon_{k} \in V_{2}\left(\epsilon_{k}\right)$. Now assume $T \in \mathfrak{l}_{i j}$ for $1 \leq i<j \leq r$. If $i \leq k$, then the joint Peirce rules imply $D_{u, \bar{v}} \epsilon_{k} \in V_{i k}$, which is a subspace of $V_{2}\left(\epsilon_{k}\right)$. If $i>k$, then $j>k$ and the joint Peirce rules imply $D_{u, \bar{k}} \epsilon_{k}=0$. For the last case, $T \in \mathfrak{l}_{i 0}$, the joint Peirce rules yield $D_{u, \bar{v}} \epsilon_{k}=0$. To sum up, this shows that $T \epsilon_{k} \in V_{2}\left(\epsilon_{k}\right)$ for all $T \in \mathfrak{p}$, which completes the proof.

We call two idempotents $\mathbf{e}, \mathbf{c} \in \mathcal{I}_{k}$ Peirce equivalent if $\pi_{k}(\mathbf{e})=\pi_{k}(\mathbf{c})$, i.e., if their Peirce 2-spaces in $V$ coincide.

We show that the Peirce varieties $\mathcal{P}_{k}$ can be imbedded $L$-equivariantly into the compact Hermitian symmetric space $X=G / Q$. Recall the Jordan-theoretic description of $X$ via projective equivalence, $X \cong\left(V \times V^{\prime}\right) / R$. The following is a crucial observation due to O. Loos; cf. [Lo94, §2.6].

Lemma 5.2. Two idempotents $\mathbf{e}, \mathbf{c} \in \mathcal{I}_{k} \subseteq V \times V^{\prime}$ are Peirce equivalent if and only if they are projectively equivalent, i.e., $V_{2}(\mathbf{e})=V_{2}(\mathbf{c})$ if and only if $\left[e: e^{\prime}\right]=\left[c: c^{\prime}\right]$.

Applying this lemma, it follows that the map

$$
\iota_{k}: \mathcal{P}_{k} \rightarrow X, U \mapsto\left[e: e^{\prime}\right] \text { for } U=V_{2}(\mathbf{e}) \text { with } \mathbf{e}=\left(e, e^{\prime}\right)
$$


is well defined and one-to-one. We may illustrate the situation in the following commutative diagram:

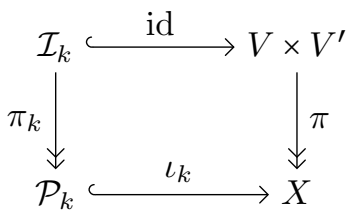

Moreover, since $h \mathbf{e}=\left(h e, h^{-\#} e^{\prime}\right)$ and $h\left[e: e^{\prime}\right]=\left[h e: h^{-\#} e^{\prime}\right]$, this diagram is also $L$-equivariant, and in particular $\iota_{k}$ is an $L$-equivariant isomorphism onto its image

$$
X_{k}:=\iota_{k}\left(\mathcal{P}_{k}\right) \subseteq X,
$$

which is a closed $L$-orbit in $X$. In this way, we have identified $r+1$ different $L$-orbits in $X$. Recalling a classical result, that there are precisely $r+1$ closed $L$-orbits in $X$, we summarize the arguments of this section.

Proposition 5.3. The closed L-orbits in $X$ are precisely the complex analytic submanifolds

$$
X_{k}=\left\{\left[e: e^{\prime}\right] \mid\left(e, e^{\prime}\right) \in \mathcal{I}_{k}\right\} \subseteq X
$$

for $k=0, \ldots, r$. The L-orbit $X_{k}$ is L-equivariantly isomorphic via $\iota_{k}$ to the Peirce variety $\mathcal{P}_{k}$.

5.2. Restriction isomorphism. In the following, we use the notation of diagram (17) and consider the restrictions of the fundamental line bundle $\mathscr{L}$ on $X$ to the closed $L$-orbits $\left(X_{k}\right)_{k=0, \ldots, r}$ that are described in Proposition 5.3. Due to Lo78, Proposition 3.1], the projection $\pi$ trivializes the line bundle $\mathscr{L}$, i.e., $\pi^{*} \mathscr{L}$ is trivial, and sections in $\mathscr{L}$ can be identified with holomorphic maps on $V \times V^{\prime}$ satisfying a certain cocycle condition, i.e.,

$$
H^{0}(X, \mathscr{L}) \cong\left\{\begin{array}{l|l}
f: V \times V^{\prime} \rightarrow \mathbb{C} \text { hol. } & \begin{array}{l}
f(x, y)=\Delta\left(x, y-y^{\prime}\right) \cdot f\left(x^{y-y^{\prime}}, y^{\prime}\right) \\
\text { for all quasi-invertible }\left(x, y-y^{\prime}\right)
\end{array}
\end{array}\right\} .
$$

In this realization, the $L$-action on sections is given by

$$
(h \cdot f)(x, y)=\chi(h) \cdot f\left(h^{-1} x, h^{\#} y\right) \quad \text { for } \quad h \in L,(x, y) \in V \times V^{\prime},
$$

where $\chi: Q \rightarrow \mathbb{C}^{\times}$is the defining character of $\mathscr{L}$. Let $\left.\mathscr{L}\right|_{X_{k}}$ denote the restriction of the line bundle to the closed $L$-orbit $X_{k} \subseteq X$. By compactness of $X_{k}$, the space $H^{0}\left(X_{k},\left.\mathscr{L}\right|_{X_{k}}\right)$ is finite dimensional. Moreover, $L$ acts irreducibly. Due to Proposition 5.3 and the commutativity of (17), we obtain the identifications

$$
\begin{aligned}
& H^{0}\left(X_{k},\left.\mathscr{L}\right|_{X_{k}}\right) \cong H^{0}\left(\mathcal{P}_{k}, \iota_{k}^{*} \mathscr{L}\right) \\
& \cong\left\{\begin{array}{l|l}
f: \mathcal{I}_{k} \rightarrow \mathbb{C} \text { hol. } & \begin{array}{l}
f\left(e, e^{\prime}\right)=\Delta\left(e, e^{\prime}-c^{\prime}\right) f\left(c, c^{\prime}\right) \\
\text { for all Peirce equivalent }\left(e, e^{\prime}\right),\left(c, c^{\prime}\right)
\end{array}
\end{array}\right\} .
\end{aligned}
$$

We note that due to Lemma 3.2, $\Delta\left(e, e^{\prime}-c^{\prime}\right)=\Delta_{\mathbf{e}}^{\prime}\left(c^{\prime}\right)$ for $\mathbf{e}=\left(e, e^{\prime}\right)$, which is the (opposite) Jordan algebra determinant defined by the idempotent $\mathbf{e}$. The main result of this section asserts that the decomposition of $H^{0}(X, \mathscr{L})$ into $L$-types is obtained by restricting the sections to the closed $L$-orbits. 
Theorem 5.4. The restriction map

$$
\rho: H^{0}(X, \mathscr{L}) \rightarrow \bigoplus_{k=0}^{r} H^{0}\left(X_{k},\left.\mathscr{L}\right|_{X_{k}}\right), f \mapsto\left(\left.f\right|_{X_{0}}, \ldots,\left.f\right|_{X_{r}}\right)
$$

is an isomorphism of L-modules.

Proof. For convenience, we set $\mathcal{H}:=H^{0}(X, \mathscr{L})$ and $\mathcal{H}_{k}:=H^{0}\left(X_{k},\left.\mathscr{L}\right|_{X_{k}}\right)$. Since the $X_{k}$ are closed $L$-orbits, each $\mathcal{H}_{k}$ is an irreducible representation of $L$, and $\rho$ is an $L$-equivariant map. To show that $\rho$ is onto, we first consider the map $f_{a}(x, y):=\Delta(x, y-a)$ for $(x, y) \in V \times V^{\prime}$ and some fixed $a \in V^{\prime}$. Applying the basic identity $\Delta(u, v) \Delta\left(u^{v}, w\right)=\Delta(u, w+v)$ of the Jordan pair determinant (see [L075, §16.11]), one immediately verifies that $f_{a}$ is an element of $\mathcal{H}$. The restriction of $f_{a}$ to $\mathcal{I}_{k}$ is given by $f_{a}\left(e, e^{\prime}\right)=\Delta_{\mathbf{e}}^{\prime}(a)$ with $\mathbf{e}=\left(e, e^{\prime}\right)$. Therefore, Lemma 3.3 implies that $\left.f_{a}\right|_{\mathcal{I}_{k}}=0$ if and only if $\operatorname{rk} a<k$. Choosing rk $a$ maximal, this shows that for each $k$, the restriction map $\rho_{k}(f):=\left.f\right|_{X_{k}}$ is non-trivial, and hence Schur's Lemma and the irreducibility of $\mathcal{H}_{k}$ implies that each $\rho_{k}$ is surjective. Due to Proposition 5.5 below, the $L$-modules $\mathcal{H}_{k}$ are mutually inequivalent. It thus follows readily that $\rho=\left(\rho_{1}, \ldots, \rho_{r}\right)$ is also surjective. However, we note that surjectivity of $\rho$ can also be proved without using the inequivalence of the $L$-modules $\mathcal{H}_{k}$. Indeed, for $\ell=0, \ldots, r$ consider the map $\rho^{\ell}: \mathcal{H} \rightarrow \mathcal{H}_{r-\ell} \oplus \cdots \oplus \mathcal{H}_{r}$. We show by induction on $\ell$ that all maps $\rho^{\ell}$ are surjective. We already know that $\rho^{0}=\rho_{r}$ is surjective. For $\ell>0$, choose $a \in V^{\prime}$ with $\operatorname{rk} a=r-\ell$. Due to Lemma 3.3. $\rho^{\ell}\left(f_{a}\right)$ is non-trivial only in the component of $\mathcal{H}_{r-\ell}$. Therefore, the $L$-module generated by $\rho^{\ell}\left(f_{\mathbf{c}}\right)$ in $\mathcal{H}_{r-\ell} \oplus \cdots \oplus \mathcal{H}_{r}$ is a non-trivial submodule of the first component, and by irreduciblibly of $\mathcal{H}_{r-\ell}$, we obtain

$$
\mathcal{H}_{r-\ell} \oplus\{0\} \oplus \cdots \oplus\{0\} \subseteq \rho^{\ell}(\mathcal{H}) .
$$

Now let $\left(f_{r-\ell}, \ldots, f_{r}\right)$ be any element in $\mathcal{H}_{r-\ell} \oplus \cdots \oplus \mathcal{H}_{r}$. By induction hypothesis, there exists an element $f \in \mathcal{H}$ with $\left.f\right|_{\mathcal{I}_{j}}=f_{j}$ for all $j>r-\ell$. Due to (20), the first component can be fixed by choosing a section $g \in \mathcal{H}$ with $\left.g\right|_{\mathcal{I}_{r-\ell}}=f_{r-\ell}-\left.f\right|_{\mathcal{I}_{r-\ell}}$ and $\left.g\right|_{\mathcal{I}_{j}}=0$ for $j>r-\ell$. Therefore, $\rho^{\ell}(f+g)=\left(f_{r-\ell}, \ldots, f_{r}\right)$, and hence $\rho^{\ell}$ is surjective. In particular, $\rho=\rho^{r}$ is surjective.

To show that $\rho$ is injective, we have to show that a section $f \in H^{0}(X, \mathscr{L})$ that vanishes along all closed $L$-orbits $\left(X_{k}\right)_{k=0, \ldots, r}$ must also vanish on all of $X$. For this we use an inductive argument showing

$$
f\left(e+c, e^{\prime}\right)=0 \quad \text { for all orthogonal } \mathbf{e}=\left(e, e^{\prime}\right), \mathbf{c}=\left(c, c^{\prime}\right) \in \mathcal{I} .
$$

From Proposition 3.4 and the fact that any element admits a completion to an idempotent, it follows that any element in $V \times V^{\prime}$ is projectively equivalent to some element of the form $\left(e+c, e^{\prime}\right)$ as in (21). Therefore, showing (21) also proves that $\rho$ is injective. As above, let $r$ be the rank of $\left(V, V^{\prime}\right)$. We prove (21) by induction on $n=r-k$ with $k=\operatorname{rk}(\mathbf{e})$. For $n=0$, the statement just reads $f\left(e, e^{\prime}\right)=0$ for all $\mathbf{e}=\left(e, e^{\prime}\right) \in \mathcal{I}_{r}$, since $c \in V_{0}(\mathbf{e})=\{0\}$. This is satisfied by the assumption $\left.f\right|_{\mathcal{I}_{r}}=0$. For $n>0$, we start a second proof by induction, namely induction on $\operatorname{rk}(\mathbf{c})$. For $\operatorname{rk}(\mathbf{c})=0$, i.e., $c=0$, we have $f\left(e, e^{\prime}\right)=0$ due to the assumption that $\left.f\right|_{\mathcal{I}_{k}}=0$. For $\operatorname{rk}(\mathbf{c}) \geq 1$, consider the decomposition $\mathbf{c}=\mathbf{c}_{1}+\mathbf{c}_{2}$ for some orthogonal idempotents $\mathbf{c}_{i}=\left(c_{i}, c_{i}^{\prime}\right)$ with $\operatorname{rk}\left(\mathbf{c}_{2}\right)=1$. Due to the cocycle condition on $f$, we obtain for any $t \in \mathbb{C}$

$$
f\left(e+c_{1}+t c_{2}, e^{\prime}\right)=\Delta\left(e+c_{1}+t c_{2},-\left(1-\frac{1}{t}\right) c_{2}^{\prime}\right) f\left(e+c_{1}+c_{2}, e^{\prime}+\left(1-\frac{1}{t}\right) c_{2}^{\prime}\right) .
$$


By orthogonality of $\mathbf{e}, \mathbf{c}_{1}$ and $\mathbf{c}_{2}$, the Jordan determinant simplifies to

$$
\Delta\left(t c_{2},-\left(1-\frac{1}{t}\right) c_{2}^{\prime}\right)=\Delta\left(c_{2},(1-t) c_{2}^{\prime}\right)=\Delta_{c}^{\prime}\left(t c_{2}^{\prime}\right)=t^{\mathrm{rk}\left(\mathbf{c}_{2}\right)}=t,
$$

and we therefore obtain

$$
f\left(e+c_{1}+t c_{2}, e^{\prime}\right)=\operatorname{tg}\left(\frac{1}{t}\right) \quad \text { with } \quad g\left(\frac{1}{t}\right):=f\left(e+c_{1}+c_{2}, e^{\prime}+\left(1-\frac{1}{t}\right) c_{2}^{\prime}\right)
$$

for some entire function $g \in \mathcal{O}(\mathbb{C})$. Since the left hand side is holomorphic in $t$, it follows that $g\left(\frac{1}{t}\right)=\alpha+\beta \frac{1}{t}$ for some $\alpha, \beta \in \mathbb{C}$. By induction hypothesis (on $n$ ), $g(0)=0$, so $\alpha=0$, and we conclude

$$
f\left(e+c_{1}+t c_{2}, e^{\prime}\right)=\beta=\text { const. }
$$

Setting $t=0$, the induction hypothesis on $\operatorname{rk}(\mathbf{c})$ yields $\beta=f\left(u_{+}+e_{+}, u_{-}\right)=0$, and hence we also obtain $f\left(e+c, e^{\prime}\right)=f\left(e+c_{2}+c_{1}, e^{\prime}\right)=0$. This finally proves (21) and completes the proof of the theorem.

The next proposition determines the highest weights of the $L$-types $H^{0}\left(X_{j},\left.\mathscr{L}\right|_{X_{j}}\right)$ and gives an explicit description of a highest weight vector. Let $\left(e_{1}, \ldots, e_{r}\right)$ be the frame of tripotents associated to the strongly orthogonal roots $\gamma_{1}, \ldots, \gamma_{r}$ (cf. Section (3.4), and let $\lambda$ be the fundamental weight associated to $\gamma_{1}$. Set $\epsilon_{k}:=\sum_{i=1}^{k} e_{i}$ for $k=0, \ldots, r$.

Proposition 5.5. For $k \in\{0, \ldots, r\}$, the L-type $H^{0}\left(X_{k},\left.\mathscr{L}\right|_{X_{k}}\right)$ has highest weight (with respect to $\Phi_{L}^{+}$)

$$
\lambda_{k}:=\lambda+\gamma_{1}+\cdots+\gamma_{k} \in(i \mathfrak{t})^{*} .
$$

In particular, the decomposition (19) is multiplicity free. Moreover, the map

$$
f_{k}(x, y):=\Delta\left(x, y-\bar{\epsilon}_{k}\right)
$$

defines a section $f_{k} \in H^{0}(X, \mathscr{L})$, the restriction of which to $X_{k}$ is a highest weight vector of $H^{0}\left(X_{k},\left.\mathscr{L}\right|_{X_{k}}\right)$. In local coordinates $\iota_{0}: V \hookrightarrow X$, the trivialization $\tilde{f}_{k}$ of $f_{k}$ is given by $\tilde{f}_{k}(x)=\Delta\left(x,-\bar{\epsilon}_{k}\right)$ for $x \in V$, and the restriction to the $\mathbb{C}$-linear span of the frame $\left(e_{1}, \ldots, e_{r}\right)$ is

$$
\tilde{f}_{k}(x)=\left(1+x_{1}\right) \cdots\left(1+x_{k}\right) \quad \text { for } \quad x=x_{1} e_{1}+\cdots+x_{r} e_{r}
$$

with $x_{1}, \ldots, x_{r} \in \mathbb{C}$.

Proof. Let $Q_{k}$ be the $L$-stabilizer of the element $\left[\epsilon_{k}: \bar{\epsilon}_{k}\right] \in X_{k}$. According to Proposition 5.3. $Q_{k}$ is also the $L$-stabilizer of the Peirce 2-space $V_{2}\left(\epsilon_{k}\right)$, and Lemma 5.1 shows that the opposite Borel subgroup $B_{L}^{\text {op }}$ (i.e., the one with Lie algebra $\mathfrak{b}_{L}^{\text {op }}:=$ $\left.\mathfrak{h} \oplus \mathfrak{n}_{L}^{-}\right)$is contained in $Q_{k}$. Therefore, to determine the weight of $H^{0}\left(X_{k},\left.\mathscr{L}\right|_{X_{k}}\right)$, it suffices to determine the character $\chi_{k}$ corresponding to the line bundle $\left.\mathscr{L}\right|_{X_{k}}$. Due to (17), this is the same character as the one corresponding to $\iota_{k}^{*} \mathscr{L}$ on $\mathcal{P}_{k}$. Hence, $\chi_{k}$ can be read from (18) and the cocycle condition which describes sections in $\iota_{k}^{*} \mathscr{L}$, namely

$$
\chi_{k}: Q_{k} \rightarrow \mathbb{C}, h \mapsto \chi(h) \cdot \Delta\left(\epsilon_{k}, \bar{\epsilon}_{k}-h^{\#} \bar{\epsilon}_{k}\right)=\chi(h) \cdot \Delta_{\epsilon_{k}}^{\prime}\left(h^{\#} \bar{\epsilon}_{k}\right),
$$

where $\chi$ is the character of $\mathscr{L}$ (restricted to $Q_{k} \subseteq L$ ). We note that $\Delta_{\epsilon_{k}}^{\prime}$ is the (opposite) Jordan algebra determinant, and one can show that $\Delta_{\epsilon_{k}}^{\prime}\left(h^{\#} \bar{\epsilon}_{k}\right)=\Delta_{\epsilon_{k}}\left(h^{-1} \epsilon_{k}\right)$, which is a more common description. Here, we prefer to use the first formula for $\chi_{k}$. Let $\mathfrak{q}_{k}$ denote the Lie algebra of $Q_{k}$. Next, we determine the derivative of $\chi_{k}$ at $e \in Q_{k}$ along an element $X \in \mathfrak{q}_{k}$. By definition, the derivative of the character $\chi$ 
of $\mathscr{L}$ is the linear functional $\lambda$. For the second term, $\tilde{\chi}_{k}(h):=\Delta\left(\epsilon_{k}, \bar{\epsilon}_{k}-h^{\#} \bar{\epsilon}_{k}\right)$, we note that

$$
\frac{\partial}{\partial y} \Delta(x, y)(z)=-\frac{1}{p} \Delta(x, y) \tau\left(x^{y}, z\right)
$$

where $p$ is defined by (8). This identity is an immediate consequence of the relation $\Delta(x, y)^{p}=\operatorname{det} B_{x, y}$ (see [Lo75, §17.3]) and the Jordan identity JP30. By a short computation we thus obtain

$$
d \tilde{\chi}_{k}(e)(X)=\frac{1}{p} \tau\left(\epsilon_{k}, X^{\#} \bar{\epsilon}_{k}\right)=\frac{1}{p} \tau\left(X \epsilon_{k}, \bar{\epsilon}_{k}\right) .
$$

Recall that the Cartan subalgebra $\mathfrak{h} \subseteq \mathfrak{q}_{k}$ decomposes into $\mathfrak{h}=\mathfrak{s}_{\mathbb{C}} \oplus \mathfrak{s}_{\mathbb{C}}^{\perp}$, where the elements $H \in \mathfrak{s}_{\mathbb{C}}^{\perp}$ have the property that $H\left(e_{j}\right)=0$ for all $j$. Therefore, $d \tilde{\chi}_{k}(e)$ vanishes along $\mathfrak{s}_{\mathbb{C}}^{\perp}$. Moreover, $\mathfrak{s}_{\mathbb{C}}$ is spanned by the elements $H_{\gamma_{j}}=D_{e_{j}, \bar{e}_{j}}$ for $j=$ $1, \ldots, r$ and by the central element $\operatorname{Id}_{V}$, and we obtain by (strong) orthogonality of the tripotents of the frame elements

$$
d \tilde{\chi}_{k}(e)\left(H_{\gamma_{j}}\right)=\frac{1}{p} \tau\left(D_{e_{j}, \bar{e}_{j}} \epsilon_{k}, \bar{\epsilon}_{k}\right)= \begin{cases}\frac{2}{p} \tau\left(e_{j}, \bar{e}_{j}\right)=2 & , \text { if } j \leq k, \\ 0 & , \text { if } j>k .\end{cases}
$$

Together with $d \chi(e)\left(\operatorname{Id}_{V}\right)=k$, it follows that $d \tilde{\chi}_{k}(e)$ coincides on $\mathfrak{h}$ with the functional $\gamma_{1}+\cdots+\gamma_{k}$, and we therefore conclude that $d \chi_{k}(e)=\lambda+\gamma_{1}+\cdots+\gamma_{k}$. Next, we consider the map $f_{k}$.

Due to the identity $\Delta(u, v) \Delta\left(u^{v}, w\right)=\Delta(u, w+v)$ it follows that $f_{k}$ satisfies the cocycle condition for sections in $H^{0}(X, \mathscr{L})$. The evaluation of $\tilde{f}_{k}(x)=f_{k}(x, 0)=$ $\Delta\left(x,-\bar{\epsilon}_{k}\right)$ at $x=x_{1} e_{1}+\cdots+x_{r} e_{r}$ is a special case of the formula $\Delta(x, y)=\prod_{i=1}^{r}(1-$ $\left.x_{i} y_{i}\right)$ with $y=y_{1} \bar{e}_{1}+\cdots+y_{r} \bar{e}_{r}$; cf. [Lo75, $\left.\S 16.15\right]$. To prove that $f_{k}$ is a highest weight vector of the $L$-type $H^{0}\left(X_{k},\left.\mathscr{L}\right|_{X_{k}}\right)$, it suffices to show that $f_{k}$ is constant on an open subset of $X_{k}$ which contains $\left[\epsilon_{k}: \bar{\epsilon}_{k}\right]$, since $B_{L}^{\text {op }} \subseteq Q_{k}$ (due to the next lemma). Indeed, $f_{k}$ is even constant on the open subset $U_{\bar{\epsilon}_{k}}=\left\{\left[x: \bar{\epsilon}_{k}\right] \mid x \in V\right\} \subseteq X$, since $f_{k}\left(x, \bar{\epsilon}_{k}\right)=\Delta(x, 0)=1$ for all $x \in V$. This completes the proof.

\section{REDUCED SPACES FOR THE $K$-ACTION}

6.1. Moment map. In this section we explicitly determine the moment map of the $K$-action on the compact Hermitian symmetric space $X=U / K$. Recall from Section 4.1 that $X$ can be realized as the coadjoint orbit $\mathcal{O}_{\lambda} \subseteq \mathfrak{u}^{*}$ with base point $i \lambda \in \mathfrak{u}^{*}$, where $-\lambda$ is the extension of the fundamental weight associated to $\gamma_{1}$. In this realization the moment map $\mu: \mathcal{O}_{\lambda} \rightarrow \mathfrak{u}^{*}$ is just the inclusion $\mathcal{O}_{\lambda} \subseteq \mathfrak{u}^{*}$, and the moment map $\mu_{\mathfrak{k}}$ corresponding to the $K$-action on $X \cong \mathcal{O}_{\lambda}$ is given by restriction to $\mathfrak{k}$, i.e., $\mu_{\mathfrak{k}}(x)=\left.\mu(x)\right|_{\mathfrak{k}}$ for all $x \in \mathcal{O}_{\lambda}$.

In the following we use a constant multiple of the Killing form $\kappa$ on $\mathfrak{u}$ to identify $\mathfrak{u}^{*}$ with $\mathfrak{u}, \mathfrak{k}^{*}$ with $\mathfrak{k}$, and coadjoint orbits with the corresponding adjoint orbits. More precisely, we use

$$
\vartheta: \mathfrak{u} \rightarrow \mathfrak{u}^{*}, X \mapsto \frac{1}{2 p} \kappa(X,-),
$$

where $p$ is defined in (8). Let $\tilde{\mu}:=\vartheta \circ \mu$ and $\tilde{\mu}_{\mathfrak{k}}:=\vartheta \circ \mu_{\mathfrak{k}}$ denote the corresponding moment maps. Then, $\tilde{\mu}_{\mathfrak{k}}=\mathrm{pr}_{\mathfrak{k}} \circ \tilde{\mu}$, where $\mathrm{pr}_{\mathfrak{k}}$ is the orthogonal projection of $\mathfrak{u}$ onto $\mathfrak{k}$ with respect to the Killing form.

Lemma 6.1. The map $\vartheta$ identifies $i \lambda \in \mathfrak{u}^{*}$ with $i \operatorname{Id}_{V} \in \mathfrak{k}$, which is central in $\mathfrak{k}$. 
Proof. Let $\eta \in \mathfrak{u}$ be the unique element determined by the relation $i \lambda(Y)=\kappa(X, Y)$ for all $Y \in \mathfrak{u}$. We have to show that $X=\frac{i}{2 p} \operatorname{Id}_{V}$. Since $\lambda$ vanishes on $\mathfrak{k}^{\perp}$, it follows that $X \in \mathfrak{k}$. In addition, the relation $\left(\operatorname{Ad}_{u}^{*} \lambda\right)(Y)=\kappa\left(X, \operatorname{Ad}_{u}^{-1} Y\right)=\kappa\left(\operatorname{Ad}_{u} X, Y\right)$ implies that the stabilizer of the coadjoint action of $U$ with respect to $\lambda$ (which is $K$ ) coincides with the stabilizer of the adjoint action of $U$ with respect to $X$. Therefore, $X$ is central in $\mathfrak{k}$, and since $\mathfrak{z}(\mathfrak{k})=\mathbb{R} \cdot i \operatorname{Id}_{V}$, we conclude that $X=c \cdot i \operatorname{Id}_{V}$ for some $c \in \mathbb{R}$. Finally, using (7), the relation $\lambda\left(H_{\gamma_{1}}\right)=-1$ with $H_{\gamma_{1}}=-D_{e_{1}, \bar{e}_{1}}$ implies

$$
i=(i \lambda)\left(-H_{\gamma_{1}}\right)=\kappa\left(c \cdot i \operatorname{Id}_{V}, D_{e_{1}, \bar{e}_{1}}\right)=2 i c \cdot \operatorname{Tr} D_{e_{1}, \bar{e}_{1}}=2 i c \cdot \tau\left(e_{1}, \bar{e}_{1}\right),
$$

and since $p=\tau\left(e_{1}, \bar{e}_{1}\right)$, this completes the proof.

As a first step, we determine the moment map on the open and dense subset $V \subseteq X$. Recall the Jordan-theoretic description of $X$ via projective equivalence, $X \cong\left(V \times V^{\prime}\right) / R$, so elements of $X$ are equivalence classes $[x: a]$ of elements in $V \times V^{\prime}$. The embedding $\iota_{0}: V \hookrightarrow X$ is given by $\iota_{0}(x)=[x: 0]$. The moment map on $X=U / K$ is by definition identified with the moment map $\tilde{\mu}_{\mathfrak{k}}$ on $\vartheta^{-1}\left(\mathcal{O}_{\lambda}\right) \subseteq \mathfrak{u}$ via the isomorphism given by $u K \mapsto \operatorname{Ad}_{u}\left(i \operatorname{Id}_{V}\right)$.

Proposition 6.2. The restriction of the moment map $\tilde{\mu}_{\mathfrak{k}}: X \rightarrow \mathfrak{k}$ of the $K$-action on $X$ to $V \subseteq X$ is given by

$$
\tilde{\mu}_{\mathfrak{k}}: V \rightarrow \mathfrak{k}, x \mapsto i\left(B_{x,-\bar{x}}^{-1}-Q_{x} B_{-\bar{x}, x}^{-1} Q_{\bar{x}}\right) .
$$

If $x=\sum_{j=1}^{k} \sigma_{j} e_{j}$ is the spectral decomposition of $x \in V$, then

$$
\tilde{\mu}_{\mathfrak{k}}(x)=i\left(\operatorname{Id}_{V}-\sum_{j=1}^{k} \frac{\sigma_{j}^{2}}{1+\sigma_{j}^{2}} D_{e_{j}, \bar{e}_{j}}\right) .
$$

Proof. Let $\pi: U \rightarrow X \cong U / K$ be the canonical projection of $U$ onto $X$ given by $u \mapsto u[0: 0]$. Then the embedding $\iota_{0}: V \hookrightarrow X$ of $V$ into $X$ admits a lift to $U$ given by

$$
\varphi: V \rightarrow U, x \mapsto u_{x}:=t_{x} \circ B_{x,-\bar{x}}^{1 / 2} \circ \tilde{t}_{\bar{x}}
$$

Indeed, since $\tilde{t}_{\bar{x}}[0: 0]=[0: \bar{x}]=[0: 0]$, we obtain

$$
\pi \circ \varphi(x)=u_{x}[0: 0]=t_{x} \circ B_{x,-\bar{x}}^{1 / 2}[0: 0]=t_{x}[0: 0]=[x: 0]=\iota_{0}(x) .
$$

Therefore, the moment map on $V \subseteq X$ is given by $\tilde{\mu}: V \rightarrow \mathfrak{u}$ with $\tilde{\mu}^{x}:=\tilde{\mu}(x)=$ $\operatorname{Ad}_{u_{x}}\left(i \mathrm{Id}_{V}\right)$. Recall that all Lie algebras are realized as vector fields on $V$ and that the adjoint action reads

$$
\left(\operatorname{Ad}_{u^{-1}} \zeta\right)(z)=d u(z)^{-1} \cdot \zeta(u(z)) .
$$

Explicitly, since $u_{x}^{-1}(z)=t_{-x} B_{x,-\bar{x}}^{1 / 2} \tilde{t}_{-\bar{x}}(z)=B_{x,-\bar{x}}^{1 / 2} z^{-\bar{x}}-x$, we obtain

$$
\begin{aligned}
\tilde{\mu}^{x}(z) & =i\left(B_{x,-\bar{x}}^{1 / 2} B_{z,-\bar{x}}^{-1}\right)^{-1} \cdot\left(i u_{x}^{-1}(z)\right) \\
& =i B_{z,-\bar{x}} z^{-\bar{x}}-i B_{z,-\bar{x}} B_{x,-\bar{x}}^{-1 / 2} x \\
& =i\left(z+Q_{z} \bar{x}-B_{z,-\bar{x}}\left(x^{-\bar{x}}\right)\right) .
\end{aligned}
$$

In the last step we used the identity $B_{x,-\bar{x}}^{-1 / 2} x=x^{-\bar{x}}$, which is obvious when $x$ is replaced by its spectral decomposition $x=\sum \sigma_{j} e_{j}$. Recall that the moment map $\tilde{\mu}_{\mathfrak{k}}$ 
of the $K$-action on $V \subseteq X$ is given by the orthogonal projection of $\tilde{\mu}^{x}$ onto $\mathfrak{k}$. The orthogonal projection yields the linear terms of the vector field $\tilde{\mu}^{x}$; therefore,

$$
\tilde{\mu}_{\mathfrak{k}}^{x}(z)=d \tilde{\mu}^{x}(0) \cdot z=i\left(z-D_{z, \bar{x}}\left(x^{-\bar{x}}\right)\right)=i\left(\operatorname{Id}_{V}-D_{x^{-\bar{x}}, \bar{x}}\right)(z) .
$$

From this, formula (23) follows with a short calculation by using the relations $B_{x, y} D_{x^{y}, z}=D_{x, z}-Q_{x} Q_{y, z}(\mathrm{JP} 30)$ and $B_{x, y} Q_{x^{y}}=Q_{x^{y}} B_{y, x}=Q_{x}$ (JP28), and formula (24) follows by using the spectral decomposition $x=\sum \sigma_{j} e_{j}$. This completes the proof.

For the extension of the result of Proposition 6.2 to all of $X$, we need the following operator.

Lemma 6.3. For $(x, a) \in\left(V, V^{\prime}\right)$, let $\Gamma_{x, a}:=B_{x, a} B_{x^{a},-\bar{x}^{\bar{a}}} B_{\bar{a}, \bar{x}}$. Then

(a) $\Gamma_{x, a}$ depends polynomially on $x, \bar{x}, a, \bar{a}$.

(b) If $[x: a]=[z: b]$, then $\Gamma_{z, b}=B_{x, a-b}^{-1} \Gamma_{x, a} B_{\bar{a}-\bar{b}, \bar{x}}^{-1}$.

(c) $\Gamma_{x, a}$ is a positive definite operator on $V$ with respect to the inner product $(-\mid-)$ defined in (4).

Mutatis mutandis, the same results hold for the adjoint operator $\Gamma_{\bar{x}, \bar{a}}:=\Gamma_{x, a}^{\#}=$ $B_{\bar{x}, \bar{a}} B_{\bar{x}^{\bar{a}},-x^{a}} B_{a, x} \in \operatorname{End}\left(V^{\prime}\right)$, where the adjoint is taken with respect to the trace form $\tau$.

Proof. The proof of (a) and (b) relies on the fact that the Bergman operator satisfies the relations $B_{u, v} B_{u^{v}, w}=B_{u, v+w}$ and $B_{w, u^{v}} B_{v, u}=B_{v+w, u}$. For (a), consider the operator $\Gamma_{x, a, y, b}^{\prime}:=B_{x, a} B_{x^{a},-y^{b}} B_{b, y}$ with $(x, a) \in\left(V, V^{\prime}\right)$ and $(y, b) \in\left(V^{\prime}, V\right)$. Then

$$
\Gamma_{x, a, y, b}^{\prime}=B_{x,-y^{b}+a} B_{b, y}=B_{x, a} B_{-x^{a}+b, y},
$$

and the first of these identities implies that $\Gamma_{x, a, y, b}^{\prime}$ is polynomial in $(x, a) \in V$, whereas the second identity implies that $\Gamma_{x, a, y, b}^{\prime}$ is polynomial in $(y, b) \in\left(V^{\prime}, V\right)$. Therefore, $\Gamma_{x, a}=\Gamma_{x, a, \bar{x}, \bar{a}}^{\prime}$ is polynomial in $x, a, \bar{x}, \bar{a}$. Part (b) is a simple application of the identities statisfied by the Bergman operators, since $[z: b]=[x: a]$ implies $z=x^{a-b}$. For (c), we first note that $\Gamma_{x, a}$ is self-adjoint with respect to $(-\mid-)$. Due to (b) and Proposition [3.4, it suffices to prove (c) for $(x, a)=(e+z, \bar{e})$ for some tripotent $e \in S$ and $z \in V_{0}(e)$. We calculate $\Gamma_{e+z, \bar{e}}$ by means of the limit $\lim _{t \rightarrow 1} \Gamma_{e+z, \overline{t e}}$ with $t \in \mathbb{R} \backslash\{1\}$. Using the relation $(e+z)^{\overline{t e}}=\frac{1}{1-t} e+z$ and the Peirce rules, it is straightforward to obtain

$$
\Gamma_{e+z, \bar{e}}=\lim _{t \rightarrow 1} \Gamma_{e+z, \overline{t e}}=\lim _{t \rightarrow 1} B_{(1-t) e,-(1-t) \bar{e}} B_{z,-\bar{z}}=B_{z,-\bar{z}} .
$$

Let $z=\sum_{i=1}^{k} \sigma_{i} e_{i}$ be the spectral decomposition of $z$, and let $V=\bigoplus_{0 \leq i \leq j \leq k} V_{i j}$ be the joint Peirce decomposition with respect to the orthogonal system of tripotents $\left(e_{1}, \ldots, e_{k}\right)$. Then by [Lo77, §3.15], the relation $B_{z,-\bar{z}} v_{i j}=\left(1+\sigma_{i}^{2}\right)\left(1+\sigma_{j}^{2}\right) v_{i j}$ holds for all $v_{i j} \in V_{i j}$, where $\lambda_{0}:=0$. This shows that $B_{z,-\bar{z}}$, and hence $\Gamma_{e+z, \bar{z}}$ is a positive definite operator on $V$.

Theorem 6.4. The moment map $\tilde{\mu}_{\mathfrak{k}}: X \rightarrow \mathfrak{k}$ of the $K$-action on $X$ is given by

$$
\tilde{\mu}_{\mathfrak{k}}([x: a])=i\left(B_{\bar{a}, \bar{x}} \Gamma_{x, a}^{-1} B_{x, a}-Q_{x} \Gamma_{\bar{x}, \bar{a}}^{-1} Q_{\bar{x}}\right) .
$$


If $(e+z, \bar{e}) \in[x: a]$ is a representative as in Proposition 3.4 and if $z=\sum_{j=1}^{k} \sigma_{j} e_{j}$ is the spectral decomposition of $z$, then

$$
\tilde{\mu}_{\mathfrak{k}}([x: a])=i\left(\operatorname{Id}_{V}-\sum_{j=1}^{k} \frac{\sigma_{j}^{2}}{1+\sigma_{j}^{2}} D_{e_{j}, \bar{e}_{j}}-D_{e, \bar{e}}\right) .
$$

Proof. For (26), it suffices to check that the right hand side (RHS) is well defined and that its restriction to $V \subseteq X$ coincides with (23). The latter part is easy to see, since for $a=0$, we obtain $B_{x, 0}=\operatorname{Id}_{V}$ and $\Gamma_{x, 0}=B_{x,-\bar{x}}$. To prove that (RHS) is well defined, let $[x: a]=[z: b]$, so $z=x^{a-b}$. Due to Lemma 6.3, $\Gamma_{z, b}=B_{x, a-b}^{-1} \Gamma_{x, a} B_{\bar{a}-\bar{b}, \bar{x}}^{-1}$, and hence

$$
\begin{aligned}
\tilde{\mu}_{\mathfrak{k}}([z: b]) & =i\left(B_{\bar{b}, \bar{z}} \Gamma_{z, b}^{-1} B_{z, b}-Q_{z} \Gamma_{\bar{z}, \bar{b}}^{-1} Q_{\bar{z}}\right) \\
& =i\left(B_{\bar{b}, \bar{x}^{\bar{a}-\bar{b}}} B_{\bar{a}-\bar{b}, \bar{x}} \Gamma_{x, a}^{-1} B_{x, a-b} B_{x^{a-b}, b}+Q_{x^{a-b}} B_{a-b, x} \Gamma_{\bar{x}, \bar{a}}^{-1} B_{\bar{x}, \bar{a}-\bar{b}} Q_{\bar{x}^{\bar{a}-\bar{b}}}\right) \\
& =i\left(B_{\bar{a}, \bar{x}} \Gamma_{x, a}^{-1} B_{x, a}-Q_{x} \Gamma_{\bar{x}, \bar{a}}^{-1} Q_{\bar{x}}\right) \\
& =\tilde{\mu}_{\mathfrak{k}}([x: a]) .
\end{aligned}
$$

We note that continuity also yields the condition $\tilde{\mu}_{\mathfrak{k}}([x: a]) \in \mathfrak{k}$. The identity (27) follows by continuity from (24), since $[x: a]=[e+z: \bar{e}]=\lim _{t \rightarrow 1}\left[\frac{1}{1-t} e+z: 0\right]$, and the spectral decomposition of $\frac{1}{1-t} e+z$ is just $\frac{1}{1-t} e+z=\frac{1}{1-t} e+\sum \sigma_{j} e_{j}$, since $z \in V_{0}(e)$.

Corollary 6.5. The image of the moment map $\tilde{\mu}_{\mathfrak{k}}$ in $\mathfrak{k}$ is given by

$$
\tilde{\mu}_{\mathfrak{k}}(X)=\left\{\begin{array}{l|l}
i\left(\operatorname{Id}_{V}-\sum_{i=1}^{r} \nu_{i} D_{e_{i}, \bar{e}_{i}}\right) & \begin{array}{l}
\left(e_{1}, \ldots, e_{r}\right) \text { frame of tripotents, } \\
\left(\nu_{1}, \ldots, \nu_{r}\right) \in[0,1]^{r}
\end{array}
\end{array}\right\} .
$$

In the last part of this section, we return to the moment map $\mu_{\mathfrak{k}}$ in its original form with image in $\mathfrak{k}^{*}$ and determine the intersection of $\mu_{\mathfrak{k}}(X)$ with the closed positive Weyl chamber $i \mathfrak{c} \subseteq \mathfrak{k}^{*}$ (with respect to $\Phi_{L}^{+}$), i.e.,

$$
\mathfrak{c}:=\left\{\alpha \in(i \mathfrak{t})^{*} \mid \alpha\left(H_{\beta}\right) \geq 0 \text { for all } \beta \in \Phi_{L}^{+}\right\} .
$$

It is a well-known result of Kirwan (cf. Ki84) that this intersection is a convex polytope. In our setting, this readily follows from the explicit description of the intersection.

Recall from Section 4.1 that real-valued functionals $\alpha \in(i \mathfrak{t})^{*}$ are identified with their complex extensions to functionals on $\mathfrak{l}$ (a zero-extension on the orthogonal complement of $i \mathfrak{t}$ ), and the restriction of $i \alpha$ to $\mathfrak{k}$ is a real-valued functional on $\mathfrak{k}$, so $i \mathfrak{c} \subseteq \mathfrak{k}^{*}$.

Theorem 6.6. The intersection of the image of the moment map $\mu_{\mathfrak{k}}$ with ic is given by

$$
\mu_{\mathfrak{k}}(X) \cap i \mathfrak{c}=\left\{i\left(\lambda+\sum_{j=1}^{r} \nu_{j} \gamma_{j}\right) \in \mathfrak{t}^{*} \mid 1 \geq \nu_{1} \geq \ldots \geq \nu_{r} \geq 0\right\}=: \Pi_{\mathfrak{s}} .
$$

In particular, this is a convex polytope.

Proof. Let $\left(e_{1}, \ldots, e_{r}\right)$ be the frame of tripotents that is associated to the maximal abelian subalgebra $\mathfrak{t} \subseteq \mathfrak{u}$ as described in Section 3.4. Consider

$$
\tilde{\Pi}_{\mathfrak{s}}:=\left\{i\left(\operatorname{Id}_{V}-\sum_{i=1}^{r} \nu_{i} D_{e_{i}, \bar{e}_{i}}\right) \mid 1 \geq \nu_{1} \geq \ldots \geq \nu_{r} \geq 0\right\} \subseteq \mathfrak{k} .
$$


We first show that $\vartheta\left(\tilde{\Pi}_{\mathfrak{s}}\right)=\Pi_{\mathfrak{s}}$ with $\vartheta$ as in (22). Indeed, Lemma 6.1 shows that $\vartheta(i \mathrm{Id})=i \lambda$, and using (9), we obtain for all $T \in \mathfrak{k}$,

$$
\begin{aligned}
\vartheta\left(i D_{e_{j}, \bar{e}_{j}}\right)(T) & =\frac{i}{2 p} \kappa\left(D_{e_{j}, \bar{e}_{j}}, T\right)=-\frac{i}{2 p} \kappa\left(T,\left[e_{j}, q_{\bar{e}_{j}}\right]\right) \\
& =-\frac{i}{2 p} \kappa\left(T e_{j}, q_{\bar{e}_{j}}\right)=\frac{i}{p} \tau\left(T e_{j}, \bar{e}_{j}\right)=-i \gamma_{j}(T) .
\end{aligned}
$$

Therefore, $\vartheta\left(i D_{e_{j}, \bar{e}_{j}}\right)=-i \gamma_{j}$, and we conclude that $\vartheta\left(\tilde{\Pi}_{\mathfrak{s}}\right)=\Pi_{\mathfrak{s}}$. Since $K$ acts transitively on the set of frames of tripotents, Corollary 6.5 implies that $\operatorname{Ad}_{K}\left(\tilde{\Pi}_{\mathfrak{s}}\right)=$ $\tilde{\mu}_{\mathfrak{k}}(X)$, and since $\vartheta$ is $K$-equivariant, this also yields

$$
\operatorname{Ad}_{K}^{*}\left(\Pi_{\mathfrak{s}}\right)=\mu_{\mathfrak{k}}(X) .
$$

As a second step, we prove that $\Pi_{\mathfrak{s}} \subseteq i \mathrm{c}$ : Theorem 5.4 and Proposition 5.5 imply that for all $\ell \in\{0,1, \ldots, r\}$ the functional $\lambda_{\ell}:=\lambda+\sum_{j=1}^{\ell} \gamma_{j} \in(i \mathfrak{t})^{*}$ is a highest weight of an $L$-type in $H^{0}(X, \mathscr{L})$, hence $i \lambda_{\ell} \in \Pi_{\mathfrak{s}} \cap i \mathfrak{c}$. Since $i \mathfrak{c}$ is convex, we conclude that

$$
\Pi_{\mathfrak{s}}=\operatorname{conv}\left\{i \lambda_{0}, \ldots, i \lambda_{r}\right\} \subseteq i \mathfrak{c} .
$$

Finally, since the closed Weyl chamber $i \mathfrak{c}$ is a fundamental domain for the $K$-action on $\mathfrak{k}^{*}$ (cf. DK00, Lemma 3.8.2]), it follows from (28) and (29) that $\mu_{\mathfrak{k}}(X) \cap i \mathfrak{c}=$ $\Pi_{\mathfrak{s}}$.

6.2. Reduced spaces. The next goal is to show that the reduced spaces are points, so we first determine the fibre $\tilde{\mu}_{\mathfrak{k}}^{-1}(T)$ of a given element $T=i\left(\operatorname{Id}_{V}-\sum_{j=1}^{k} \nu_{j} D_{e_{j}, \bar{e}_{j}}\right) \epsilon$ $\mathfrak{k}$.

Lemma 6.7. Let $\left(e_{1}, \ldots, e_{k}\right)$ and $\left(c_{1}, \ldots, c_{\ell}\right)$ be two systems of orthogonal tripotents, and let $\nu_{1}<\nu_{2}<\cdots<\nu_{k}$ and $\mu_{1}<\mu_{2}<\cdots<\mu_{\ell}$ be non-zero real numbers. Then

$$
\sum_{i=1}^{k} \nu_{i} D_{e_{i}, \bar{e}_{i}}=\sum_{j=1}^{\ell} \mu_{j} D_{c_{j}, \bar{c}_{j}} \Longleftrightarrow k=\ell \text { and } \nu_{i}=\mu_{i}, e_{i} \approx c_{i} \text { for all } i .
$$

Here, $e_{i} \approx c_{i}$ means that $e_{i}$ and $c_{i}$ induce the same Peirce decompositions, i.e., $V_{m}\left(e_{i}\right)=V_{m}\left(c_{i}\right)$ for $m \in\{0,1,2\}$.

Proof. For convenience, we set $A:=\sum_{i=1}^{k} \nu_{i} D_{e_{i}, \bar{e}_{i}}$ and $B:=\sum_{j=1}^{\ell} \mu_{j} D_{c_{j}, \bar{c}_{j}}$. Let $V=\oplus_{0 \leq i \leq j \leq r} V_{i j}$ be the joint Peirce decomposition with respect to the orthogonal family $\left(e_{1}, \ldots, e_{k}\right)$. Then, the Peirce rules imply $A x_{i j}=\left(\nu_{i}+\nu_{j}\right) x_{i j}$ for $x_{i j} \in V_{i j}$, where we also set $\nu_{0}:=0$. Therefore, $V$ decomposes into eigenspaces of $A$, and the eigenspace of the highest eigenvalue, namely $2 \nu_{k}$, is $V_{2}\left(e_{k}\right)$. In the same way we obtain a decomposition of $V$ into eigenspaces of $B$, and the eigenspace of the highest eigenvalue $2 \mu_{\ell}$ is $V_{2}\left(c_{\ell}\right)$. Assuming $A=B$, we therefore obtain $\nu_{k}=\mu_{\ell}$ and $V_{2}\left(e_{k}\right)=V_{2}\left(c_{\ell}\right)$. Since Peirce 2-spaces corresponding to tripotents uniquely determine the whole Peirce decomposition, this also implies $V_{m}\left(e_{k}\right)=V_{m}\left(c_{\ell}\right)$ for $m \in\{0,1,2\}$, and hence $D_{e_{k}, \bar{e}_{k}}=D_{c_{\ell}, \bar{c}_{\ell}}$. Therefore, the assumption $A=B$ is reduced to $\sum_{i=1}^{k-1} \nu_{i} D_{e_{i}, \bar{e}_{i}}=\sum_{j=1}^{\ell-1} \mu_{j} D_{c_{j}, \bar{c}_{j}}$, and the statement follows by induction.

Theorem 6.8 (Reduced spaces). Let $\tilde{\mu}_{\mathfrak{k}}: X \rightarrow \mathfrak{k}$ be the moment map of the $K$ action on $X$, and let $T \in \tilde{\mu}_{\mathfrak{k}}(X)$, i.e., $T=\tilde{\mu}_{\mathfrak{k}}\left(\left[e_{0}+z: \bar{e}_{0}\right]\right)$ for some tripotent $e_{0} \in S$ and $z \in V_{0}\left(e_{0}\right)$. Let $z=\sum_{j=1}^{k} \sigma_{j} e_{j}$ be the spectral decomposition of $z$. 
(a) The fibre of $T$ with respect to the moment map is given by

$$
\tilde{\mu}_{\mathfrak{k}}^{-1}(T)=\left\{\left[c_{0}+\sum_{j=1}^{k} \sigma_{j} c_{j}: \bar{c}_{0}\right] \mid \begin{array}{l}
c_{j} \in S, c_{j} \approx e_{j} \\
\text { for all } j=0, \ldots, k
\end{array}\right\} .
$$

(b) The stabilizer subgroup $K_{T}$ of $T$ consists of those elements leaving the Peirce spaces of the joint Peirce decomposition $V=\oplus V_{i j}$ with respect to the orthogonal system $\left(e_{1}, \ldots, e_{k}, e\right)$ invariant.

(c) The reduced space $\tilde{\mu}_{\mathfrak{k}}^{-1}(T) / K_{T}$ is a point.

Proof. Recall from Proposition 3.4 that any element of $X$ can be represented as $\left[c_{0}+w: \bar{c}_{0}\right]$ for some tripotent $c_{0}$ and $w \in V_{0}\left(c_{0}\right)$. Let $w=\sum_{i=1}^{\ell} \tau_{i} c_{i}$ be the spectral decomposition of $w$. Now assume that $\tilde{\mu}_{\mathfrak{k}}\left(\left[e_{0}+z: \bar{e}_{0}\right]\right)=\tilde{\mu}_{\mathfrak{k}}\left(\left[c_{0}+w: \bar{c}_{0}\right]\right)$. Then, Theorem 6.4 and Lemma 6.7 imply that $k=\ell$ and $\sigma_{j}^{2} /\left(1+\sigma_{j}^{2}\right)=\tau_{j}^{2} /\left(1+\tau_{j}^{2}\right), c_{j} \approx e_{j}$ for all $j=0, \ldots, k$. Moreover, since the identity between $\sigma_{j}$ and $\tau_{j}$ is solved only for $\sigma_{j}= \pm \tau_{j}$, and $\sigma_{j}, \tau_{j}$ are assumed to be positive, this proves (a). For (b), we note that $\operatorname{Ad}_{k} D_{e_{i}, \bar{e}_{i}}=D_{k e_{j}, \overline{k e}_{j}}$, and hence Lemma 6.7 implies that $k \in K$ stabilizes $T$ if and only if $e_{j} \approx k e_{j}$ for all $j$. Since the Peirce spaces of the joint Peirce decomposition corresponding to $\left(e_{0}, e_{1}, \ldots, e_{k}\right)$ can be described by intersections of the Peirce spaces $V_{m}\left(e_{j}\right)$ with $m \in\{0,1,2\}, j=0, \ldots, k$, and, conversely, the Peirce spaces $V_{m}\left(e_{j}\right)$ are given by direct sums of joint Peirce spaces, this proves (b). For (c), we have to show that $K_{T}$ acts transitively on $\tilde{\mu}_{\mathfrak{k}}^{-1}(T)$. Due to (a), this is equivalent to the statement that $K_{T}$ conjugates any orthogonal systems $\left(e_{0}, \ldots, e_{k}\right)$ and $\left(c_{0}, \ldots, c_{k}\right)$ of tripotents with $e_{j} \approx c_{j}$ for all $j$. This follows from the fact $([$ Lo77, §5.9]) that $K$ acts transitively on the set of frames of tripotents: since each $e_{j}$ and $c_{j}$ can be decomposed further into orthogonal primitive tripotents to obtain frames, and since $\mathrm{rk} e_{j}=\mathrm{rk} c_{j}$, there exists an element $k \in K$ mapping $c_{j}$ onto $e_{j}$ for all $j$. Since $c_{j} \approx e_{j}, k$ preserves the Peirce spaces $V_{m}\left(e_{j}\right)=V_{m}\left(c_{j}\right)$. By the same argument as for (b), it follows that $k \in K_{T}$.

\section{BRANCHING LAWS}

Before we turn to the problem of decomposing the spaces $H^{0}\left(X, \mathscr{L}^{k}\right)$ under the group $K$, we recall some notions from the theory of Hamiltonian actions of compact Lie groups. For a more thorough treatment, we refer to [GS82] and [Sj95].

Let $K$ temporarily denote an arbitrary compact connected Lie group which acts holomorphically on the connected compact Kähler manifold $(M, \Omega)$ in a Hamiltonian fashion, and let $\tau: M \rightarrow \mathfrak{k}^{*}$ be the moment map for the action. Assume that $\mathcal{L} \rightarrow M$ is a prequantum line bundle for $(M, \Omega)$. Then $K$ acts holomorphically on $\mathcal{L}$ as bundle isomorphisms, and this action extends to an action of the universal complexification, $K^{\mathbb{C}}$, of $K$. We recall two notions of stability for the $K$-action on $M$. First, we have the set

$$
M_{s s}(\mathcal{L}):=\left\{m \in M \mid s(m) \neq 0 \text { for some } k \in \mathbb{N} \text {, and } s \in H^{0}\left(M, \mathcal{L}^{k}\right)^{K}\right\}
$$

of algebraically semistable points. Second, we have the set

$$
M_{s s}=\left\{m \in M \mid \overline{K^{\mathbb{C}} \cdot m} \cap \tau^{-1}(0) \neq \varnothing\right\}
$$


of analytically semistable points. In fact, the identity

$$
M_{s s}(\mathcal{L})=M_{s s}
$$

holds (cf. [Sj95, Thm. 2.18]).

We now turn to our particular setting, where $K$ again is the stabilizer in $U$ of $e Q \in X$. Let $\mathcal{O}_{\xi}^{K}$ be a coadjoint orbit in $\mathfrak{k}^{*}$ through an integral $i \xi \in \mathfrak{t}^{*} \subseteq \mathfrak{k}^{*}$, which we view as a symplectic manifold when equipped with the Kostant-Kirillov symplectic form $\omega_{\xi}^{K}$. Let $\overline{\mathcal{O}_{\xi}^{K}}$ denote $\mathcal{O}_{\xi}^{K}$ equipped with the symplectic form $-\omega_{\xi}^{K}$ and with the reverse complex structure, i.e., the sheaf of holomorphic functions on $\overline{\mathcal{O}_{\xi}^{K}}$ is the sheaf of antiholomorphic functions on $\mathcal{O}_{\xi}^{K}$. If $\mathscr{L}_{\xi}$ is the prequantum line bundle for $\mathcal{O}_{\xi}^{K}$, let $\overline{\mathscr{L}_{\xi}^{*}}$ denote the line bundle over $\mathcal{O}_{\xi}^{K}$ where the fibre over $x$ is given by the complex-antilinear functionals $\left(\mathscr{L}_{\xi}\right)_{x} \rightarrow \mathbb{C}$. Then $\overline{\mathscr{L}_{\xi}^{*}}$ is a holomorphic prequantum line bundle for $\left(\overline{\mathcal{O}_{\xi}^{K}},-\omega_{\xi}^{K}\right)$.

Now consider the product space $X \times \overline{\mathcal{O}_{\xi}^{K}}$. Let $p_{1}$ and $p_{2}$ denote the projections onto $X$ and $\overline{\mathcal{O}_{\xi}^{K}}$, respectively. For $k \in \mathbb{N}$, we equip $X \times \overline{\mathcal{O}_{\xi}^{K}}$ with the symplectic form $p_{1}^{*}\left(k \omega_{\lambda}\right)-p_{2}^{*} \omega_{\xi}^{K}$. The diagonal action of $K$ on $X \times \overline{\mathcal{O}_{\xi}^{K}}$, when equipped with this symplectic form, is then holomorphic and Hamiltonian with moment map

$$
\mu_{\mathfrak{k}}^{k, \xi}(x, y):=\mu_{\mathfrak{k}}^{k}(x)-y, \quad(x, y) \in X \times \overline{\mathcal{O}_{\xi}^{K}} .
$$

Put

$$
\mathcal{L}(\xi, k):=p_{1}^{*} \mathscr{L}^{k} \otimes p_{2}^{*} \overline{\mathscr{L}_{\xi}^{*}} .
$$

Then $\mathcal{L}(\xi, k)$ is a holomorphic prequantum line bundle for $\left(\left(X \times \overline{\mathcal{O}_{\xi}^{K}}\right), p_{1}^{*}\left(k \omega_{\lambda}\right)-\right.$ $\left.p_{2}^{*} \omega_{\xi}^{K}\right)$, and the $K$-action lifts to a holomorphic action on $\mathcal{L}(\xi, k)$.

From Theorem 6.8 (c) we immediately conclude the following result.

Proposition 7.1. For every $k \in \mathbb{N}$, the group $K$ acts transitively on $\left(\mu_{\mathfrak{k}}^{k, \xi}\right)^{-1}(0) \subseteq$ $X \times \overline{\mathcal{O}_{\xi}^{K}}$.

Proposition 7.2. For every $\xi \in(i \mathfrak{t})^{*}$ and $k \in \mathbb{N}$, the dimension of the space $H^{0}(X \times$ $\left.\overline{\mathcal{O}_{\xi}^{K}}, \mathcal{L}(\xi, k)\right)^{K}$ is at most one.

Proof. First of all, by [Sj95, Thm. 2.18],

$$
H^{0}\left(X \times \overline{\mathcal{O}_{\xi}^{K}}, \mathcal{L}(\xi, k)\right)^{K} \cong H^{0}\left(\left(X \times \overline{\mathcal{O}_{\xi}^{K}}\right)_{s s}, \mathcal{L}(\xi, k)\right)^{K} .
$$

By the definition of $\left(X \times \overline{\mathcal{O}_{\xi}^{K}}\right)_{s s}$ any $K$-invariant section, also being $K^{\mathbb{C}}$-invariant, is uniquely determined by its values on $\left(\mu_{\mathfrak{k}}^{k, \xi}\right)^{-1}(0)$. By Proposition 7.1 such a section is in fact determined by its value at some given point in $\left(\mu_{\mathfrak{k}}^{k, \xi}\right)^{-1}(0)$. This finishes the proof.

Lemma 7.3. The integral points in $\mu_{\mathfrak{k}}^{k}(X) \cap i \mathfrak{c}$ are precisely the points $k \lambda+m_{1} \gamma_{1}+$ $\cdots+m_{r} \gamma_{r}$, where $k, m_{i} \in \mathbb{Z}$ such that $k \geq m_{1} \geq \ldots \geq m_{r} \geq 0$.

Proof. Clearly, every point $k \lambda+m_{1} \gamma_{1}+\cdots+m_{r} \gamma_{r}$ with $k \geq m_{1} \geq \ldots \geq m_{r} \geq 0$ is integral. For the converse inclusion, let $\xi \in \mu_{\mathfrak{k}}^{k}(X) \cap i \mathfrak{c}$. Then $\xi=k \lambda+x_{1} \gamma_{1}+\cdots+x_{r} \gamma_{r}$ for some $x_{i} \in \mathbb{R}$ satisfying $k \geq x_{1} \geq \ldots \geq x_{r} \geq 0$. If $\xi$ is integral, then $x_{1} \gamma_{1}+\cdots+x_{r} \gamma_{r}$ 
is also integral. From the argument at the end of the proof of Lemma 2 in [Sc69], it then follows that $x_{i} \in \mathbb{Z}$ for $i=1, \ldots, r$. This finishes the proof.

Theorem 7.4. The space $H^{0}\left(X, \mathscr{L}^{k}\right)$ decomposes under $L$ as

$$
H^{0}\left(X, \mathscr{L}^{k}\right)=\bigoplus_{k \geq m_{1} \geq \ldots \geq m_{r} \geq 0} W_{(k \lambda, \boldsymbol{m})},
$$

where $W_{(k \lambda, \boldsymbol{m})}$ is the irreducible L-representation with highest weight $k \lambda+\sum_{i=1}^{r} m_{i} \gamma_{i}$.

Proof. By Theorem 1.1 and Lemma 7.3. only representations of the form $W_{(k \lambda, m)}$ can occur in $H^{0}\left(X, \mathscr{L}^{k}\right)$, and by Proposition 7.2 they can at most have multiplicity one. Thus, it suffices to prove that every such representation actually does occur. For this, we note that every $\boldsymbol{m} \in \mathbb{N}_{0}^{r}$ satisfying the condition $k \geq m_{1} \geq \ldots \geq m_{r} \geq 0$ can be written uniquely as $\boldsymbol{m}=\sum_{j=1}^{k} \boldsymbol{m}(j)$, with $\boldsymbol{m}(j)=\left(m_{1}(j), \ldots, m_{r}(j)\right) \in \mathbb{N}_{0}^{r}$ satisfying $1 \geq m_{1}(j) \geq \ldots \geq m_{r}(j) \geq 0$. By Theorem [5.4 and Proposition 5.5, for each such $\boldsymbol{m}(j)$ the irreducible $L$-representation $W_{(\lambda, \boldsymbol{m}(j))}$ occurs in $H^{0}(X, \mathscr{L})$. Let $s_{j} \in H^{0}(X, \mathscr{L})$ be an $L$-highest weight vector for the representation $W_{(\lambda, \boldsymbol{m}(j))}$. Then $s_{1} \cdots s_{k} \in H^{0}\left(X, \mathscr{L}^{k}\right)$ is an $L$-highest weight vector of weight $k \lambda+\sum_{i=1}^{r} m_{i} \gamma_{i}$. Hence, the representation $W_{(k \lambda, \boldsymbol{m})}$ occurs in $H^{0}\left(X, \mathscr{L}^{k}\right)$.

\section{The OKounkov Body}

In this section we return to the Okounkov body $\Delta\left(\mathscr{L}, N_{L}^{+}, v\right)$ (cf. (16)). The main result is an identification of $\Delta\left(\mathscr{L}, N_{L}^{+}, v\right)$ with the moment polytope $\Pi_{\mathfrak{s}}$. We also prove that the semigroup $S\left(\mathscr{L}, N_{L}^{+}, v\right)$ (cf. (15)) is finitely generated.

Let $\boldsymbol{m} \in \mathbb{N}_{0}^{r}$ satisfy $1 \geq m_{1} \geq \ldots \geq m_{r} \geq 0$, and let $s_{\boldsymbol{m}} \in H^{0}(X, \mathscr{L})$ be an $L$-highest weight vector with highest weight $\lambda+m_{1} \gamma_{1}+\cdots+m_{r} \gamma_{r}$.

Proposition 8.1. The semigroup $S\left(\mathscr{L}, N_{L}^{+}, v\right)$ is generated by the elements (1, $\left.v\left(s_{\boldsymbol{m}}\right)\right)$ with $1 \geq m_{1} \geq \ldots \geq m_{r} \geq 0$.

Proof. Let $s \in H^{0}\left(X, \mathscr{L}^{k}\right)$ be an $N_{L}^{+}$-invariant vector. Then $s$ can be written as a linear combination $s=\sum_{i} s_{i}$ of $L$-highest weight vectors in $H^{0}\left(X, \mathscr{L}^{k}\right)$ corresponding to distinct highest weights. Hence, by Proposition 4.1, $v\left(s_{i}\right) \neq v\left(s_{j}\right)$ for $i \neq j$. It follows immediately from the definition of $v$ that $v(s)=v\left(s_{i}\right)$ for some $s_{i}$, namely the $s_{i}$ with smallest $v\left(s_{i}\right)$. We can thus, without loss of generality, assume that $s$ is an $L$-highest weight vector. By Theorem 7.4 the weight of $s$ can then be written as $k \lambda+\sum_{i=1}^{r} m_{i} \gamma_{i}$ with $k \geq m_{1} \geq \ldots \geq m_{r} \geq 0$. As in the proof of Theorem 7.4, $\boldsymbol{m}:=\left(m_{1}, \ldots, m_{r}\right)$ can be written uniquely as $\boldsymbol{m}=\sum_{j=1}^{k} \boldsymbol{m}(j)$, with $\boldsymbol{m}(j)=\left(m_{1}(j), \ldots, m_{r}(j)\right) \in \mathbb{N}_{0}^{r}$ satisfying $1 \geq m_{1}(j) \geq \ldots \geq m_{r}(j) \geq 0$. Let $s_{j} \in H^{0}(X, \mathscr{L})$ be an $L$-highest weight vector with weight $\lambda+\sum_{i=1}^{r} m_{i}(j) \gamma_{i}$. Then $s_{1} \cdots s_{k} \in H^{0}\left(X, \mathscr{L}^{k}\right)$ is an $L$-highest weight vector of weight $k \lambda+\sum_{i=1}^{r} m_{i} \gamma_{i}$. Since the decomposition under $L$ is multiplicity free, $s$ is a scalar multiple of $s_{1} \cdots s_{k}$. Hence, by (13), $v(s)=v\left(s_{1}\right)+\cdots+v\left(s_{k}\right)$. This finishes the proof.

Corollary 8.2. The Okounkov body $\Delta\left(\mathscr{L}, N_{L}^{+}, v\right)$ is the convex hull of the points $\left(1, v\left(s_{\boldsymbol{m}}\right)\right)$ with $1 \geq m_{1} \geq \ldots \geq m_{r} \geq 0$.

Let $\mathcal{P} \subseteq \mathfrak{t}^{*}$ denote the weight lattice of $\mathfrak{t}$. Define the moment semigroup

$$
S\left(\mathscr{L}, N_{L}^{+}\right):=\left\{(k, i \xi) \in \mathbb{N} \times \mathcal{P} \mid \xi \text { is the highest weight of some } W_{(k \lambda, \boldsymbol{m})}\right\} .
$$


We now construct a morphism of semigroups, $\Lambda$, from $S\left(\mathscr{L}, N_{L}^{+}, v\right)$ to $S\left(\mathscr{L}, N_{L}^{+}\right)$. As already observed in the proof of Proposition 8.1 two weight vectors $s_{1}, s_{2} \epsilon$ $H^{0}\left(X, \mathscr{L}^{k}\right)$ with distinct weights cannot have the same value under $v$. Now, let $(k, a) \in S\left(\mathscr{L}, N_{L}^{+}, v\right)$. Since the decomposition of $H^{0}\left(X, \mathscr{L}^{k}\right)$ is multiplicity free there exists a unique (up to scalar multiples) weight vector $s \in H^{0}\left(X, \mathscr{L}^{k}\right)^{N_{L}^{+}}$with $v(s)=a$. We therefore define

$$
\Lambda: S\left(\mathscr{L}, N_{L}^{+}, v\right) \rightarrow S\left(\mathscr{L}, N_{L}^{+}\right)
$$

by putting $\Lambda((k, a)):=(k, i \xi)$, where $\xi$ is the weight of $s$. Now, if $(k, a),(\ell, b) \in$ $S\left(\mathscr{L}, N_{L}^{+}, v\right)$, where $a=v(s), b=v(t)$ for highest weight vectors $s \in H^{0}\left(X, \mathscr{L}^{k}\right), t \in$ $H^{0}\left(X, \mathscr{L}^{\ell}\right)$ of highest weight $\xi$ and $\xi^{\prime}$, respectively, then $s t \in H^{0}\left(X, \mathscr{L}^{k+\ell}\right)$ is a highest weight vector of weight $\xi+\xi^{\prime}$ and $v(s t)=v(s)+v(t)=a+b$. Hence,

$$
\Lambda((k+\ell, a+b))=\left(k+\ell, i \xi+i \xi^{\prime}\right)=\Lambda((k, a))+\Lambda((\ell, b)),
$$

i.e., $\Lambda$ is a morphism of semigroups. In fact, $\Lambda$ is given by

$$
\Lambda\left(\left(k,\left(a_{1}, \ldots, a_{n}\right)\right)\right)=\left(k, i\left(k \lambda+\sum_{j=1}^{n} a_{j} \alpha_{j}\right)\right)
$$

for all $\left(k,\left(a_{1}, \ldots, a_{n}\right)\right) \in S\left(\mathscr{L}, N_{L}^{+}, v\right)$ (cf. Proposition 4.1).

Remark 8.3. The morphism $\Lambda$ was introduced by Okounkov in Ok96 in a slightly different (but more general) setting.

Proposition 8.4. The map $\Lambda: S\left(\mathscr{L}, N_{L}^{+}, v\right) \rightarrow S\left(\mathscr{L}, N_{L}^{+}\right)$is an isomorphism of semigroups.

Proof. We first prove that $\Lambda$ is injective. For this, assume that for some $a \neq b$ we have $\Lambda((k, a))=\Lambda((k, b))$. If $s, t \in H^{0}\left(X, \mathscr{L}^{k}\right)^{N_{L}^{+}}$are highest weight vectors with $v(s)=a$ and $v(t)=b$, respectively, then $s$ and $t$ are linearly independent highest weight vectors of the same weight. This would, however, contradict the multiplicity-freeness of the decomposition of $H^{0}\left(X, \mathscr{L}^{k}\right)$ under $L$.

For the surjectivity, we note that, again by multiplicity-freeness, every highest weight $\xi$ of some $W_{(k \lambda, m)}$ is the weight of a unique (up to scalar multiples) highest weight vector $s \in H^{0}\left(X, \mathscr{L}^{k}\right)^{N_{L}^{+}}$. Then $\Lambda((k, v(s)))=(k, i \xi)$.

Let $E\left(\mathscr{L}, N_{L}^{+}, v\right) \subseteq \mathbb{R} \times \mathbb{R}^{n}$ be the $\mathbb{R}$-linear subspace generated by $S\left(\mathscr{L}, N_{L}^{+}, v\right)$, and let $E\left(\mathscr{L}, N_{L}^{+}\right) \subseteq \mathbb{R} \times \mathfrak{t}^{*}$ be the $\mathbb{R}$-linear subspace generated by $S\left(\mathscr{L}, N_{L}^{+}\right)$. We extend $\Lambda$ to a unique linear map $E\left(\mathscr{L}, N_{L}^{+}, v\right) \rightarrow E\left(\mathscr{L}, N_{L}^{+}\right)$, namely $\left(x_{0},\left(x_{1}, \ldots, x_{n}\right)\right)$ $\mapsto\left(x_{0}, i\left(x_{0} \lambda+\sum_{j=1}^{n} x_{j} \alpha_{j}\right)\right)$ (cf. 30), and we let $\Lambda$ also denote this extension.

Let $C\left(\mathscr{L}, N_{L}^{+}\right) \subseteq E\left(\mathscr{L}, N_{L}^{+}\right)$be the closed convex cone generated by $S\left(\mathscr{L}, N_{L}^{+}\right)$, and put

$$
\Delta\left(\mathscr{L}, N_{L}^{+}\right):=C\left(\mathscr{L}, N_{L}^{+}\right) \cap\left(\{1\} \times \mathfrak{t}^{*}\right) .
$$

Clearly, we can identify $\Delta\left(\mathscr{L}, N_{L}^{+}\right)$with $\Pi_{\mathfrak{s}}$ by

$$
\Delta\left(\mathscr{L}, N_{L}^{+}\right)=\left\{(1, x) \mid x \in \Pi_{\mathfrak{s}}\right\} .
$$

Using this identification, Proposition 8.4 readily yields the following identification of the Okounkov body $\Delta\left(\mathscr{L}, N_{L}^{+}, v\right)$ with the moment polytope $\Pi_{\mathfrak{s}}$. 
Theorem 8.5. The map $\Lambda: E\left(\mathscr{L}, N_{L}^{+}, v\right) \rightarrow E\left(\mathscr{L}, N_{L}^{+}\right)$restricts to a bijection

$$
\Delta\left(\mathscr{L}, N_{L}^{+}, v\right) \rightarrow \Pi_{\mathfrak{s}} .
$$

Remark 8.6. The problem of constructing polyhedral Okounkov bodies has been addressed both in the setting of group actions (such as in Ok96), as well as in the case (developed in LM09]) when the semigroup is defined by the values of all sections. Few positive results in this direction are known, however. It is known to work for torus-equivariant line bundles over toric varieties (cf. [LM09]). As examples in the setting of homogeneous spaces under the action of a complex reductive group, we would like to mention [Ok98] and, more recently, Ka11].

\section{ACKNOWLEDGEMENT}

We would like to thank Joachim Hilgert and Harald Upmeier for stimulating discussions on these topics.

\section{REFERENCES}

[Be00] Bertram, W., The geometry of Jordan and Lie structures, Lecture Notes in Mathematics. 1754. Berlin: Springer. xvi, 269 p., 2000 MR.1809879(2002e:17041)

[DK00] Duistermaat, J.J., Kolk, J.A.C. Lie groups, Universitext. Springer-Verlag, Berlin, 2000 MR.1738431 (2001j:22008)

[Fu98] Fulton, W., Intersection theory, Second Edition, Springer-Verlag, Berlin, 1998 MR.1644323 (99d:14003)

[GS82] Guillemin, V. and Sternberg, S., Geometric quantization and multiplicities of group representations, Invent. Math. 67 (1982), 515-538 MR664118 (83m:58040)

[HC56] Harish-Chandra, Representations of semisimple Lie groups. VI. Integrable and squareintegrable representations, Amer. J. Math. 78 (1956), 564-628 MR0082056 (18:490d)

[Ka11] Kaveh, K., Crystal bases and Newton-Okounkov bodies, preprint, arxiv.org/abs/1101.1687v1

[Ki84] Kirwan, F., Convexity properties of the moment mapping. III., Invent. Math. 77 (1984), 3, 547-552 MR759257 (86b:58042b)

[Ko08] Kobayashi, T., Multiplicity-free theorems of the restrictions of unitary highest weight modules with respect to reductive symmetric pairs, Representation theory and automorphic forms, Progr. Math. 255, 45-109, Birkhäuser Boston, Boston, MA, 2008 MR.2369496 (2008m:22024)

[LM09] Lazarsfeld, R., Mustaţă, M., Convex bodies associated to linear series, Ann. Sci. Éc. Norm. Supér. 42 (2009), 783-835 MR2571958 (2011e:14012)

[Lo75] O. Loos, Jordan pairs, Lecture Notes in Mathematics. 460. Berlin-Heidelberg-New York, Springer-Verlag. XVI, 218 p. 1975 MR0444721 (56:3071)

[Lo77] O. Loos, Bounded symmetric domains and Jordan pairs, Lecture Notes, University of Irvine (1977)

[Lo78] O. Loos, Homogeneous algebraic varieties defined by Jordan pairs, Monatsh. Math. 86 (1978/79), no. 2, 107-129 MR516835(80i:17022)

[Lo91] O. Loos, Diagonalization in Jordan Pairs, Journal of Algebra 143, 252-268 (1991) MR:1128659 (92m:17050)

[Lo94] O. Loos, Decomposition of projective spaces defined by unit-regular Jordan pairs, Communications in Algebra (22) 10 (1994), 3925-3964 MR:1280101(95h:17036)

[Ok96] Okounkov, A., Brunn-Minkowski inequality for multiplicities, Invent. Math. 125 (1996), no. 3, 405-411 MR1400312(99a:58074)

[Ok98] Okounkov, A., Multiplicities and Newton polytopes, Kirillov's seminar on representation theory, Amer. Math. Soc. Transl. Ser. 2, 181, 231-244, Amer. Math. Soc., Providence, RI, 1998 MR.1618759 (99e:20058)

[Sa80] Satake, I., Algebraic structures of symmetric domains, Kanô Memorial Lectures, 4. Iwanami Shoten, Tokyo; Princeton University Press, Princeton, N.J., 1980 MR.591460 (82i:32003) 
[Sc69] Schmid, W., Die Randwerte holomorpher Funktionen auf hermitesch symmetrischen Räumen, Invent. Math. 9 (1969/1970), 61-80 MR0259164(41:3806)

[Sj95] Sjamaar, R., Holomorphic slices, symplectic reduction and multiplicities of representations, Ann. of Math. (2) 141 (1995), no. 1, 87-129 MR.1314032 (96a:58098)

[Up86] Upmeier, H., Jordan algebras and harmonic analysis on symmetric spaces, Amer. J. Math. 108 (1986), no. 1, 1-25 (1986) MR821311 (87e:32047)

FAKultät FÜr Elektrotechnik, Informatik und Mathematik, Institut für Mathematik, Universität Paderborn, Warburger Str. 100, 33098 Paderborn, Germany

E-mail address: bschwarz@math.upb.de

Mathematisches Institut, Georg-August-Universität Göttingen, Bunsenstrasse 3-5, D-37073 Göttingen, Germany

E-mail address: hseppaen@uni-math.gwdg.de 